\title{
Universal correlation functions in rank 1 SCFTs
}

\author{
Simeon Hellerman, ${ }^{a}$ Shunsuke Maeda, ${ }^{a}$ Domenico Orlando, ${ }^{c, b}$ Susanne Reffert ${ }^{b}$ and \\ Masataka Watanabe ${ }^{a}$ \\ ${ }^{a}$ Kavli Institute for the Physics and Mathematics of the Universe, The University of Tokyo, \\ 5-1-5 Kashiwanoha, Kashiwa, Chiba 277-8583, Japan \\ ${ }^{b}$ Albert Einstein Center for Fundamental Physics, Institute for Theoretical Physics, \\ University of Bern, Sidlerstrasse 5, Bern CH-3012, Switzerland \\ ${ }^{c}$ INFN sezione di Torino and Arnold-Regge Center, \\ via Pietro Giuria 1, Turin 10125, Italy \\ E-mail: Simeon.Hellerman@ipmu.jp, shunsuke.maeda@ipmu.jp, \\ domenico.orlando@to.infn.it, sreffert@itp.unibe.ch, \\ masataka.watanabe@ipmu.jp
}

ABSTRACT: Carrying to higher precision the large- $\mathcal{J}$ expansion of [1], we calculate to all orders in $1 / \mathcal{J}$ the power-law corrections to the two-point functions $\mathcal{Y}_{n} \equiv|x-y|^{2 n \Delta_{\mathcal{O}}}$ $\cdot\left\langle\mathcal{O}^{n}(x) \overline{\mathcal{O}}^{n}(y)\right\rangle$ for generators $\mathcal{O}$ of Coulomb branch chiral rings in four-dimensional $\mathcal{N}=$ 2 superconformal field theories. We show these correlators have the universal large- $n$ expansion

$$
\log \left(\mathcal{Y}_{n}\right) \simeq \mathcal{J} \mathbf{A}+\mathbf{B}+\log (\Gamma(\mathcal{J}+\alpha+1))
$$

where $\mathcal{J} \equiv n \Delta_{\mathcal{O}}$ is the total $R$-charge of $\mathcal{O}^{n}$, the $\mathbf{A}$ and $\mathbf{B}$ are theory-dependent coefficients, $\alpha$ is the coefficient of the Wess-Zumino term for the Weyl $a$-anomaly, and the $\simeq$ denotes equality up to terms exponentially small in $\mathcal{J}$. Our methods combine the structure of the Coulomb-branch EFT with the supersymmetric recursion relations. However, our results constrain the power-law corrections to all orders, even for non-Lagrangian theories to which the recursion relations do not apply. For the case of $\mathcal{N}=2 \mathrm{SQCD}$, we also comment on the nature of the exponentially small corrections, which can be calculated to high precision in the double-scaling limit recently discussed by Bourget et al. in [2]. We show the exponentially small correction is consistent with the interpretation of the EFT breaking down due to the propagation of massive BPS particles over distances of order of the infrared scale $|x-y|$.

Keywords: Effective Field Theories, Extended Supersymmetry, Global Symmetries, Nonperturbative Effects

ARXIV EPRINT: 1804.01535 


\section{Contents}

1 Introduction 1

2 Diagramatics and quantization of the EFT 6

2.1 Setup 7

2.2 Normalization of the observables 8

$2.3 \alpha$-dependence of the observables $\quad 9$

2.4 Examples of diagrams 11

2.5 Universality for power law corrections versus nonuniversality for exponential $\begin{array}{ll}\text { corrections } & 14\end{array}$

3 Lagrangian theories $\quad 14$

3.1 Derivation of the $\mathcal{J}^{-m}$ corrections in Lagrangian theories 14

$\begin{array}{lll}3.2 & \text { Examples } & 16\end{array}$

4 Universal theory-independence of the $\mathcal{J}^{-m}$ corrections 18

$\begin{array}{lll}4.1 & \text { Initial comments } & 18\end{array}$

$\begin{array}{lll}4.2 & \text { Ultraviolet regulators with marginal couplings } & 18\end{array}$

$\begin{array}{ll}4.3 & \text { Universal polynomials } \\ \end{array}$

5 Universal EFT behavior compared with $S^{4}$ localization 20

6 Discussion $\quad 24$

$\begin{array}{lr}\text { A Solving the recurrence equation } & 26\end{array}$

B $\mathcal{N}=2$ supersymmetrization of the Weyl anomaly action 28

C Nonexistence of higher-derivative $F$-terms on conformally flat space $\quad 33$

D $\mathcal{N}=2$ superconformal gauge dynamics with ghost hypermultiplets $\quad 38$

$\begin{array}{ll}\text { E Saddle point value of the classical action } & 41\end{array}$

$\begin{array}{lll}F & \text { Numerics } & 46\end{array}$

\section{Introduction}

Dealing analytically with strongly coupled theories is difficult. However, working in a sector of fixed large quantum number $\mathcal{J}$ associated to a global symmetry leads to important simplifications. Starting from a strongly coupled conformal field theory (CFT), it allows us 
to write down an approximately scale-invariant effective field theory (EFT) in which most terms are suppressed by inverse powers of $\mathcal{J}$ [3]. The low-energy physics is governed by one or several Goldstone fields which encode the quantum fluctuations around the fixedcharge ground state. The resulting large-quantum-number expansion is very sensitive to the vacuum structure of the theory. In the non-supersymmetric cases of the critical $O(n)$ vector model $[3,4]$ and the $\mathrm{SU}(n)$ matrix models $[5,6]$, there is a unique fixed-charge ground state which is homogeneous in space and the anomalous dimension of the lowest operator of charge $\mathcal{J}$ comes with a leading $\mathcal{J}^{3 / 2}$ scaling. The large- $R$-charge expansion of the $\mathcal{N}=2$ superconformal $W=\Phi^{3}$ theory which has no moduli space displays the same properties and the same leading $\mathcal{J}^{3 / 2}$ scaling, which signals the spontaneous breaking of supersymmetry (susy) [3]. Things are very different for superconformal field theories (SCFTs) with a nontrivial moduli space. The moduli space of vacua implies a degenerate spectrum when the curvature of the manifold on which the SCFT lives vanishes, and consequently the curvature is always relevant in the large-quantum-number expansion. The leading $\mathcal{J}$ behavior of the conformal dimension is $\Delta_{\mathcal{J}} \sim+1 \cdot|\mathcal{J}|^{1}[1,7]$.

In theories without a small loop-suppressing parameter, in all cases where it can be checked against other methods, such as Monte Carlo simulations on the lattice [8], the conformal bootstrap [9-14] (and references therein), and exact supersymmetric methods [1, 7, 15-18], the large-quantum-number expansion converges very well to the correct answer.

In this paper we focus on the last of these, carrying to higher order the previous results of [1] in an $\mathcal{N}=2$ superconformal gauge theory in four spacetime dimensions. In [1], the $1 / \mathcal{J}$ expansion of correlation functions of chiral primary operators of dimension $\Delta$ and $R$-charge $\mathcal{J}=\Delta_{\text {total }}=n \Delta_{\mathcal{O}}$ were calculated, where $\mathcal{O}$ is a generator of the holomorphic coordinate ring of a one-complex dimensional Coulomb branch. ${ }^{1}$ We compute all the terms in the asymptotic expansion, and get an explicit universal result for any theory with a onedimensional Coulomb branch, even for models without marginal couplings. Where we can compare our results with results from localization techniques, we find beautiful agreement and can even see the leading non-universal corrections from numerical data.

The EFT of the Coulomb branch was used in [1] to estimate the two-point function

$$
|x-y|^{2 n \Delta_{\mathcal{O}}}\left\langle(\mathcal{O}(x))^{n}(\overline{\mathcal{O}}(y))^{n}\right\rangle \equiv \mathcal{Y}_{n}
$$

The $\mathcal{Y}_{n}$ are defined as correlation functions,

$$
\mathcal{Y}_{n}=|x-y|^{2 n \Delta_{\mathcal{O}}} \frac{Z_{n}}{Z_{0}}
$$

where $Z_{n}$ is the path integral with insertions of the operators and $Z_{0}$ is the path integral function without insertions. Up to the Weyl transformation of the insertions and the Weyl anomaly of the partition function itself, the path integral is the same on any conformally

\footnotetext{
${ }^{1}$ In cases where the conformal dimension $\Delta_{\mathcal{O}}$ is fractional, there may be interesting oscillatory corrections to our asymptotic formula with a period set by the denominator of $\Delta_{\mathcal{O}}$. It would be interesting to explore this question but for purposes of the present paper we simply choose $n$ such that $\mathcal{J}=n \Delta_{\mathcal{O}}$ is an integer. See $[19,20]$ for related work.
} 
flat space. In particular the expectation values $\mathcal{Y}$ transform covariantly under Weyl transformations, with the Weyl anomaly canceling when one divides $Z_{n}$ by $Z_{0}$. It is convenient to perform some calculations in the conformal frame of the sphere $S^{4}$, in which we can compare our results with those of supersymmetric localization, e.g. [15].

Translating to the conventions of [15], the absolute normalization of the path integral with and without insertions is defined as

$$
\begin{aligned}
& Z_{0} \equiv \exp \left\{q_{0}\right\}, \\
& Z_{n} \equiv \exp \left\{q_{n}\right\}, \\
& \mathcal{Y}_{n} \equiv|x-y|^{2 n \Delta_{\mathcal{O}}} 16^{-n} G_{2 n}=|x-y|^{2 n \Delta_{\mathcal{O}}} \exp \left\{q_{n}-q_{0}\right\}
\end{aligned}
$$

Indeed, the quantity $Z_{n} \equiv \exp \left\{q_{n}\right\} \equiv Z_{0} \mathcal{Y}_{n}$ is a particularly natural object in our way of computing the two-point function, as it can be thought of as a path integral with sources, which can be computed with relative ease in the EFT of the Coulomb branch.

In [1], the expansion of $q_{n}$ was carried to order $\log (\mathcal{J})$, yielding the result

$$
\begin{aligned}
\log \left(\mathcal{Y}_{n}\right) & =q_{n}-q_{0}=\mathcal{J} \log (\mathcal{J})+n \log \left(\mathbf{N}_{\mathcal{O}}\right)+\left(\alpha+\frac{1}{2}\right) \log (\mathcal{J})+\mathcal{O}\left(\mathcal{J}^{0}\right) \\
& =\log (\mathcal{J} !)+n \log \left(\mathbf{N}_{\mathcal{O}}\right)+\alpha \log (\mathcal{J})+\mathcal{O}\left(\mathcal{J}^{0}\right)
\end{aligned}
$$

where $\alpha$ is the coefficient of the Wess-Zumino term for the Weyl $a$-anomaly. The constant $\mathbf{N}_{\mathcal{O}}$ is a normalization constant that can be absorbed into the normalization of $\mathcal{O}$ itself, though for theories with a marginal coupling the operator $\mathcal{O}$ has dimension $\Delta=2$ and there is a natural normalization of $\mathcal{O}$ traceable to its relation with the actual marginal operator which is its descendant.

In this note we shall use the EFT of the Coulomb branch directly to calculate the large- $\mathcal{J}$ expansion of $q_{n}$ up to and including order $1 / \mathcal{J}$. From there, we make a number of observations about the subleading corrections to the large- $\mathcal{J}$ behavior of $q_{n}$.

Our main result is that the correlators for any theory with a one-dimensional Coulomb branch (whether it has a marginal coupling constant or not) have a universal large- $\mathcal{J}$ behavior controlled by the formula

$$
\begin{aligned}
q_{n} & \simeq \mathcal{J} \log (\mathcal{J})+\left(\alpha+\frac{1}{2}\right) \log (\mathcal{J})+(\mathbf{A}-1) \mathcal{J}+\mathbf{B}+\log \sqrt{2 \pi}+\sum_{m \geq 1} \frac{\hat{K}_{m}(\alpha)}{\mathcal{J}^{m}} \\
& \simeq \mathcal{J} \mathbf{A}+\mathbf{B}+\log [\Gamma(\mathcal{J}+\alpha+1)],
\end{aligned}
$$

where $\mathbf{A}$ and $\mathbf{B}$ are theory-dependent coefficients. The $\simeq$ indicates the presence of nonuniversal corrections that are exponentially small in $\mathcal{J}$. The simplicity and universality of the large- $\mathcal{J}$ behavior is special to the case of a Coulomb branch chiral ring of dimension 1. For a Coulomb branch of dimension $\geq 2$ the operator of lowest $U(1)$ is not generally unique, and the behavior of the two-point function of powers of a given generator may be much more complicated. In the one-dimensional case, the correlators can be computed to 
all orders in $\mathcal{J}^{-1}$ in the Coulomb branch effective action, which has the simple form:

$$
\begin{aligned}
& S_{\text {eff }}=\int \sqrt{|g|} d^{4} x \mathcal{L}_{\text {eff }}, \\
& \mathcal{L}_{\text {eff }}=\mathcal{L}_{\text {kin }}+\mathcal{L}_{\text {super }-\mathrm{WZ}}+\mathcal{L}_{\text {sources }}+\mathcal{L}_{\text {D-terms }} .
\end{aligned}
$$

where $\mathcal{L}_{\text {kin }}$ is a free kinetic term for a vector multiplet, given in (E.6); the source term is the negative logarithm of the operator insertions,

$$
\mathcal{L}_{\text {sources }} \equiv-n \log [\mathcal{O}] \delta\left(x-x_{\mathcal{O}}\right)-n \log [\overline{\mathcal{O}}] \delta\left(x-x_{\overline{\mathcal{O}}}\right)
$$

and $\mathcal{L}_{\text {super-WZ }}$ is the supersymmetrized Wess-Zumino term for the Weyl a-anomaly and the $\mathrm{U}(1)_{\mathrm{R}}$-symmetry, of which the relevant details are collected in (B.21), (B.22)-(B.28).

In the case of $\mathcal{N}=2$ supersymmetric quantum chromodynamics (SQCD) with four flavors we can estimate the leading corrections using numerical results from localization and we find that

$$
\left.\log \left(\mathcal{Y}_{n}\right)\right|_{\text {localization }} ^{\mathrm{SQCD}}-\left.\log \left(\mathcal{Y}_{n}\right)\right|_{\mathrm{EFT}} ^{\mathrm{SQCD}} \approx 1.6 e^{-\pi \sqrt{\mathcal{J} /(2 \operatorname{Im} \tau)}}=1.6 e^{-\sqrt{\pi \lambda} / 2}
$$

with high accuracy in the double scaling limit $\mathcal{J} \rightarrow \infty, \operatorname{Im} \tau \rightarrow \infty$ with $\mathcal{J} / \operatorname{Im} \tau=\lambda /(2 \pi)$ fixed as suggested in [2].

The outline of our reasoning is as follows:

- $\mathcal{N}=2$ superconformal invariance is sufficiently restrictive to forbid any possible higher-derivative $F$-terms in the EFT of the Coulomb branch, for theories of rank one. As a result, the entire Wilsonian action on the Coulomb branch is given by the tree-level effective kinetic term, the supersymmetrized Wess-Zumino term for the spontaneously broken Weyl invariance, and unknown $D$-terms which do not affect correlation functions of chiral primaries.

- The F-term content of the EFT only depends on the $a$-anomaly coefficient of the underlying CFT, which we parametrize as in [1], following the normalization in AnselmiFreedman-Grisaru-Johansen [21] (AEFJ)

$$
\begin{aligned}
\alpha & \equiv 2(\Delta a)^{[\mathrm{AEFJ}]}, \\
\Delta a & \equiv a_{\mathrm{CFT}}-a_{\mathrm{EFT}}, \\
a^{[\mathrm{AEFJ}]} & \equiv \frac{5}{24} \frac{a}{a_{\mathrm{U}(1) \text { vector multiplet }}} .
\end{aligned}
$$

It follows that all the correlators, regardless of the details, depend only on $\alpha$ in some theory-independent formula, modulo terms that are either affine in $\mathcal{J}$, coming from the normalization of the external operator and the sphere partition function or nonperturbatively small in $\mathcal{J}$, coming from the breakdown of the EFT.

- In terms of $\alpha$, the order $n^{-m}$ term in $q_{n}$ can be expressed as

$$
\left.q_{n}\right|_{\mathcal{O}\left(n^{-m}\right)}=\frac{K_{m}(\alpha)}{n^{m}}
$$


where $K_{m}(\alpha)$ is a polynomial defined by quantizing the effective theory of the Coulomb branch with Wess-Zumino coefficient $\alpha$, regularized and renormalized to preserve the spontaneously broken superconformal symmetry, see section 2 .

- For values of $\alpha$ that can be realized as superconformal gauge theories with a marginal coupling, the theory has to obey recursion relations, which lead to "effective recursion relations" for the power-law terms, which are algebraic rather than differential, because these terms must be independent of $\tau$ and $\bar{\tau}$, because of point the absence of higher-derivative $F$-terms. Using the recursion relations we can compute all the polynomials $K_{m}(\alpha)$. This leads to a unique formula for all the power-law corrections, for any value of $\alpha$, for theories that obey the effective recursion relations

$$
K_{m}(\alpha) \equiv \Delta_{\mathcal{O}}^{-m} \hat{K}_{m}^{[\text {univ }]}(\alpha),
$$

where the $\hat{K}_{m}^{[\text {univ] }}$ satisfy

$$
\frac{\mathrm{d} \hat{K}_{m+1}^{[\text {univ }]}(\alpha)}{\mathrm{d} \alpha}=-m \hat{K}_{m}^{[\text {univ] }}(\alpha)
$$

and are the coefficient of $\mathcal{J}^{-m}$ in the asymptotic expansion of $\log \Gamma(\mathcal{J}+\alpha+1)$ :

$$
\left.\hat{K}_{m}^{[\text {univ] }}(\alpha) \equiv \hat{P}_{m+1}^{[\text {univ] }}(\alpha) \equiv \log \Gamma(\mathcal{J}+\alpha+1)\right|_{\mathcal{O}\left(\mathcal{J}^{-m}\right)}=\frac{(-1)^{m+1}}{m(m+1)} B_{m+1}(\alpha+1),
$$

where $B_{m+1}$ is the Bernoulli polynomial of degree $m+1$, see section 3 .

- Since unitarity does not appear to be a logical necessity for the validity of the recursion relations, we argue that the existence of an infinite series of $\mathcal{N}=2$ superconformal $\mathrm{SU}(2)$ gauge theories with nonunitary matter, marginal gauge coupling, and distinct values of $\alpha$, proves that $\hat{K}_{m}(\alpha)$ is universally completely determined by the existence of this series. In other words, for any value of $\alpha$ the power-law corrections can be shown to obey the effective recursion relations, to all orders in $\mathcal{J}$ (but not nonperturbatively) and

$$
\hat{K}_{m}(\alpha)=\hat{K}_{m}^{[\text {univ] }}(\alpha)=\frac{(-1)^{m+1}}{m(m+1)} B_{m+1}(\alpha+1), \quad \forall m \geq 1,
$$

for any theory with a one-dimensional Coulomb branch, whether it has a marginal coupling constant or not. This is discussed in section 4 .

- We can do a simple consistency check by calculating the classical terms, without reference to any completion, unitary or nonunitary, with or without marginal coupling. We find that the $\alpha^{m+1} / \mathcal{J}^{m}$ term agrees with our proven formula.

- We can also check against direct calculations in the case of $\mathcal{N}=2 \mathrm{SQCD}$, where the correlators can be computed directly by localization. We find fantastic agreement and the precision is limited only by the omission of instanton corrections. We can 
remedy that by considering the limit suggested in [2], where we find sufficiently precise agreement that we can even see the leading nonuniversal correction, which strongly suggests an interpretation in terms of virtual Bogomol'nyi-Prasad-Sommerfield (BPS) dyons propagating over macroscopic distances, see section 5 .

The plan of this paper is as follows. In section 2 we discuss the building blocks of the Feynman diagrams which appear for the expansion of the two-point functions at order $1 / \mathcal{J}$, discussing the basic setup in section 2.1, the normalization of the observables in section 2.2 and the $\alpha$-dependence of the observables in section 2.3. In section 2.4, we give concrete examples of diagrams appearing at order $n^{0}$ and $n^{-1}$. In section 2.5, we discuss the universality for power-law corrections and the nonuniversality for exponential corrections. In section 3.1, we derive the $\mathcal{J}^{-m}$ corrections in Lagrangian theories, using recursion relations to determine all correlation functions of Coulomb branch chiral primaries for $\mathcal{N}=2$ sCFTs with a marginal coupling $\tau$. In section 3.2, we treat the concrete examples of Abelian gauge theory without matter, $\mathcal{N}=4$ super Yang-Mills (SYM) with $G=s u(2)$ and $\mathcal{N}=2$ superQCD with $G=s u(2)$ and $N_{\mathrm{F}}=4$. In section 4 , we argue that our result for the power-law corrections should apply to any value of $\alpha$, for Coulomb-branch chiral-primary correlators in any rank-one theory with any value of $\alpha$, whether or not it has a marginal coupling. In section 4.2, we discuss manifestly $\mathcal{N}=2$ superconformal ultraviolet (UV) regulators with marginal couplings which are constructed by adding "ghost" hypermultiplets with reversed spin-statistics which allow us to regulate any one-dimensional Coulomb-branch EFT with $\alpha$-coefficient satisfying $\alpha \in \frac{3}{2}-\frac{4}{3} \mathbb{Z}$. In section 4.3, we use this fact to write the power-law corrections for $\alpha \in \frac{3}{2}-\frac{4}{3} \mathbb{Z}$. In section 5 we compare the universal EFT behavior with the results from $S^{4}$ localization, finding an excellent agreement. In section 6 , we close with a brief discussion of our findings. A lot of the technical details of the above discussion is relegated to the appendix. The solution to the recurrence relations is detailed in appendix $\mathrm{A}$. The $\mathcal{N}=2$ supersymmetrization of the Weyl anomaly action is given in appendix B. The nonexistence of higher-derivative $F$-terms on conformally flat space is argued in appendix $\mathrm{C}$. The $\mathcal{N}=2$ superconformal gauge dynamics with ghost hypermultiplets which is used in section 4 is explained in appendix D. The saddle point value of the classical action is shown in appendix E. Finally, in appendix F, the numerics for the comparison to the localization result are given.

\section{Diagramatics and quantization of the EFT}

As in $[1,3,7]$, we consider the effective theory of the Coulomb branch, as an effective theory in which conformal invariance is treated as an exact nonlinearly realized dynamical symmetry, and $\mathcal{N}=2$ Weyl invariance as an exact symmetry of the dynamical fields and background fields together.

As explained in appendix $\mathrm{C}$, rank one theories are special in this regard, as the effective theory admits no superconformally invariant $F$-terms beyond the kinetic term and superWess-Zumino (wz) terms, respecting the $\mathcal{N}=2$ superconformal symmetry. All higherderivative terms allowed by the symmetries are $D$-terms. We can treat our theory as a 
Wilsonian effective action with a cutoff $\Lambda$ satisfying

$$
E_{\mathrm{IR}} \ll \Lambda \ll|\phi| .
$$

We can then compute quantum effects by regularizing and renormalizing the theory with local counterterms that remove all dependence on the cutoff scale $\Lambda$.

The absence of superconformal $F$-terms means that any counterterms consistent with the symmetries must be $D$-terms. Since two-point functions can in principle be computed by supersymmetric localization, however, they are necessarily independent of $D$-terms. It follows that all power-law $1 / n^{m}$ corrections to the logarithm of the correlator are necessarily independent of the details of the microscopic theory, depending only on the $\alpha$-coefficient.

\subsection{Setup}

Begin by representing the correlator as

$$
\mathcal{Y}_{n}=|x-y|^{2 n \Delta_{\mathcal{O}}} Z_{0}^{-1} \times Z_{n}
$$

where

$$
Z_{n} \equiv \exp \left\{q_{n}\right\}
$$

is the path integral with sources $-\mathcal{J} \log \left(\phi_{\text {hol }}\right)$ and $-\mathcal{J} \log \left(\bar{\phi}_{\text {hol }}\right)$ (with $\left.\mathcal{J}=n \Delta_{\mathcal{O}}\right)$ inserted at $x$ and $y$, respectively. The sources can also be taken to be smeared over a scale $\epsilon=$ $O\left(\Lambda^{-1}\right)$ if desired, though this makes no difference to the result in the end, as the field $\phi$ is nonsingular with respect to itself and the limit $\epsilon \rightarrow 0$ is nonsingular.

Here we have taken $\phi_{\text {hol }}$ to be normalized such that $\phi^{\Delta_{\mathcal{O}}}=\mathcal{O}$ exactly. This is the superfield that we take to be holomorphic in the background fields, if one is to turn on any background fields such as a marginal coupling. For instance, in a Lagrangian theory, $\phi_{\text {hol }}$ is the superfield whose effective kinetic term is

$$
\left.\mathcal{L}_{\text {kinetic }}=-i \times(\text { const. }) \times \int d^{4} \theta_{\mathcal{N}=2} \tau \Phi_{\text {hol }}^{2}+\text { (h.c. }\right) .
$$

For purposes of quantizing the effective theory of the Coulomb branch, it is more convenient to work in terms of the field $\phi_{\text {unit }} \equiv \sqrt{\operatorname{Im}(\tau)}$, whose kinetic term is

$$
\begin{aligned}
\mathcal{L}_{\text {kinetic }} & \left.=(\text { const. }) \times \int d^{4} \theta_{\mathcal{N}=2} \Phi_{\text {unit }}^{2}+\text { (h.c. }\right) \\
& =\left|\partial \phi_{\text {unit }}\right|^{2}+\text { fermion kinetic }+ \text { gauge kinetic }
\end{aligned}
$$

in Euclidean signature. In general, when we write $\phi$ without a subscript indicating the normalization, we shall always be referring to the field $\phi_{\text {unit }}$. More generally, the relationship between $\phi_{\text {hol }}$ and $\phi_{\text {unit }}$ can be written as

$$
\phi_{\text {hol }}=\mathbf{N}_{\mathcal{O}} \phi_{\text {unit }} .
$$

In terms of the unit-kinetic-term superfield, the correlation functions of $\phi$ are

$$
\mathcal{Y}_{n}=Z_{0}^{-1} \times Z_{n}=\mathbf{N}_{\mathcal{O}}^{2 \mathcal{J}} \times Z_{n}^{\text {(unit) }},
$$

where $Z_{n}^{(\text {unit })}$ is just the path integral with insertions of the unit normalized field,

$$
Z_{n}^{(\text {unit })} \equiv\left\langle\phi_{\text {unit }}^{n \Delta_{\mathcal{O}}}(x) \phi_{\text {unit }}^{n \Delta_{\mathcal{O}}}(y)\right\rangle
$$




\subsection{Normalization of the observables}

As discussed in [1], no superconformal effective term can contribute to orders higher than $\mathcal{J}^{0}$ in the expansion of the correlation function. The wZ terms arising both for the Weyl symmetry and $\mathrm{U}(1) R$-charge are needed to compensate the difference between the anomaly coefficients of the underlying CFT and the EFT of the Coulomb branch. They cannot be written as superconformal terms in superspace, because they explicitly break both the Weyl symmetry and $R$-symmetry of the action. We must therefore write the wz terms while being very careful and explicit about their normalization.

We start with the wZ-term of the action as given in Komargodski-Schwimmer (KS) [22] (abbreviated henceforth with the superscript $K S$ ):

$$
\begin{aligned}
S_{\mathrm{WZ}}^{[\mathrm{KS}]}=-\Delta a^{[\mathrm{KS}]} \int d^{4} x \sqrt{-g}\left[\tau E_{4}^{[\mathrm{KS}]}+\left(4\left(R^{\mu \nu}-\frac{1}{2} R g^{\mu \nu}\right) \nabla_{\mu} \tau \nabla_{\nu} \tau\right.\right. \\
\left.\left.-2(\nabla \tau)^{2}\left(2 \square \tau-(\nabla \tau)^{2}\right)\right)\right] .
\end{aligned}
$$

In a Coulomb branch EFT, there is also a mismatch of the $\mathrm{U}(1)_{R}$ gravitational anomaly coefficients between the EFT and the full underlying CFT, proportional to the Weyl $a$ anomaly mismatch [1]. This anomaly mismatch can only be canceled by a gravitational Green-Schwarz mechanism involving the Goldstone mode $\beta$ of the spontaneously broken $\mathrm{U}(1)_{R}$. Under supersymmetry the dilaton and axion form a chiral multiplet $\tau+i \beta$. For a rank-one theory there is a unique candidate for the axiodilaton. In terms of the $\phi_{\text {hol }}$ coordinate, the complex axiodilaton $\tau+i \beta$ of $[22-24]$ is

$$
\tau+i \beta=-\log (\phi / \mu) .
$$

If we want to specify how our dilaton depends on background fields, we need to specify whether we are talking about the holomorphic dilaton, unit dilaton, or something else.

The holomorphic axiodilaton is defined as [24]

$$
(\tau+i \beta)_{\text {holo }}=-\log \left(\phi_{\text {holo }} / \mu\right),
$$

while the unit-normalized axiodilaton is

$$
(\tau+i \beta)_{\mathrm{unit}}=-\log \left(\phi_{\mathrm{unit}} / \mu\right)
$$

where the two differ by

$$
(\tau+i \beta)_{\text {holo }}=(\tau+i \beta)_{\mathrm{unit}}-\log \mathbf{N}_{\mathcal{O}}
$$

The quantity $\mathbf{N}_{\mathcal{O}}$ depends on the background fields (such as complex marginal couplings $\tau)$ in a nonholomorphic way, and therefore we must keep track of it if we are trying to do things like compute the un-normalized correlators $\exp \left\{q_{n}\right\} \equiv Z \times \mathcal{Y}_{n}$ as a function of $\tau, \bar{\tau}$. For constant $\tau, \bar{\tau}$, only the overall normalization of $\exp \left\{q_{n}\right\}$ is affected by the difference between $(\tau+i \beta)_{\text {unit }}$ and $(\tau+i \beta)_{\text {holo }}$, because the undifferentiated axiodilaton 
enters the action of [24] only through the Euler density. However even apart from this, it is valuable to keep track of the difference, because one may want to compute, for instance, in backgrounds with position-dependent marginal couplings, in which case $\mathbf{N}_{\mathcal{O}}$ becomes position-dependent and its gradients enter the effective action.

In the present paper, we will only ever consider constant gauge coupling $\tau$, and therefore we will work in terms of the unit-normalized axiodilaton, taking care to add the extra term to the action of [24]:

$$
\mathcal{L}_{\mathrm{BEO}}\left[(\tau+i \beta)_{\text {holo }}\right]=(\Delta a)^{[\mathrm{KS}]} \times \log \left(\mathbf{N}_{\mathcal{O}}\right) E_{4}+\mathcal{L}_{\mathrm{BEO}}\left[(\tau+i \beta)_{\mathrm{unit}}\right],
$$

and $\mathcal{L}_{\mathrm{BEO}}$ is given in appendix $\mathrm{B}$. When we refer to the super-dilaton without specifying, we will always be referring to the unit-normalized rather than holomorphic dilaton, as the unit-normalized axiodilaton is the more natural object from the point of view of the large- $\mathcal{J}$ expansion.

In terms of the unit-normalized chiral superfield

$$
\phi \equiv \phi_{\text {unit }} \equiv \mu \exp (-\tau-i \beta) \equiv \mu \exp \left(-(\tau+i \beta)_{\text {unit }}\right),
$$

the full effective action for the path integral with sources is

$$
\begin{aligned}
& S_{\text {eff }}=\int \sqrt{|g|} d^{4} x \mathcal{L}_{\text {eff }}, \\
& \mathcal{L}_{\text {eff }}=\mathcal{L}_{\text {kin }}+\mathcal{L}_{\text {super-WZ }}+\mathcal{L}_{\text {sources }}+\mathcal{L}_{\text {D-terms }},
\end{aligned}
$$

as mentioned earlier in (1.6). The $D$-terms will not affect our considerations at all, as we are computing observables invariant under some subset of the supersymmetry, and therefore unaffected by $D$-terms.

We also note that in a more general Coulomb branch, of rank more than 1, we would expect higher-derivative $F$-terms, of the type studied in [25-28]. In a theory of rank 1 , as we show in appendix $\mathrm{C}$, there are no superconformally invariant higher-derivative $F$ terms at all. It is this simplification that permits the extraordinarily detailed calculation of correlation functions to all orders in $1 / n$ that we are able to perform in the present article.

We wish to emphasize particularly that the absence of higher-derivative $F$-terms, means that the action (2.16) is in effect an almost UV-complete action: there are no ultraviolet divergences to any order in $1 / \mathcal{J}$ perturbation theory, which affect the correlation function. Or more precisely, for a superconformally invariant regulator, there are no UV-divergences in $1 / \mathcal{J}$ perturbation theory affecting the protected correlation functions; for a non-superconformally-invariant regulator, any UV divergences will be proportional to powers of $|\Lambda| /|\phi|$, and can be subtracted in a canonical way according to the criterion of restoring superconformal invariance of the quantum effective action. We will now use this almost-UV completeness of the F-term sector of the EFT, to derive an all-orders $1 / \mathcal{J}$ expansion for the chiral primary two-point functions.

\section{$2.3 \quad \alpha$-dependence of the observables}

We can read off the form of the $\alpha$-dependence from the form of the action as written in terms of $\phi_{\text {unit }}$. Modulo $D$-terms, the only terms in the action are of order $|\phi|^{2}, \mathcal{J} \log |\phi|$, 
and $|\phi|^{0} \alpha$. If we define $\hat{\alpha} \equiv \alpha / \mathcal{J}$, then the whole action, written in terms of $\hat{\alpha}$ and $\mathcal{J}$, is strictly of order $\mathcal{J}^{1}$, modulo logarithms of $\mathcal{J}$. Thus $\mathcal{J}^{-1}$ becomes a uniform loop-counting parameter of the theory: the parameter $\mathcal{J}$ only occurs together with $1 / \hbar$, so long as we write the action in terms of $\mathcal{J}$ and $\hat{\alpha}$, the power of parameter $\mathcal{J}$ exactly counts the number of loops in a diagram:

$$
(\mathcal{J}-\text { scaling of a diagram }) \propto \mathcal{J}^{[1-(\text { number of loops })]} F(\hat{\alpha}) .
$$

Since we are computing the partition function and counting the source terms as part of the action itself, the diagrams we are computing are vacuum diagrams, with no "external" lines.

Concretely, the classical solution for $\phi$ is of order $\mathcal{J}^{1 / 2}$ and if we split the classical solution into $\phi=\phi_{\text {classical }}+\phi_{\text {fluc }}$, then we can decompose the free + source + the super-WZ into vacuum expectation value (VEV) and fluctuations; each vertex with $f$ fluctuations scales as $\mathcal{J}^{1-f / 2}$ at fixed $\hat{\alpha}$, and by the usual counting, a connected vacuum diagram, after contracting all fluctuation lines, must have scaling $\mathcal{J}^{1 \text {-(number of loops) }}$. As usual we ignore $D$-terms, of which the correlators are independent.

To find the $\mathcal{J}$-scaling at fixed $\alpha$ of a diagram, simply turn the $\hat{\alpha}$ 's back into $\alpha / \mathcal{J}$ 's, which gives an extra factor of $1 / \mathcal{J}$ for each $\alpha$-vertex. This gives

$$
\begin{aligned}
& (\mathcal{J}-\text { scaling of a diagram }) \propto \mathcal{J}^{-m}, \\
& m \equiv(\# \text { of loops })+(\# \text { of } \alpha-\text { vertices })-1 .
\end{aligned}
$$

From formula (2.18) two important properties of correlation functions in the Coulombbranch EFT as a function of $\alpha$ are immediately clear:

- The term $\hat{K}_{m} / \mathcal{J}^{m} \in q_{n}$ is a polynomial in $\alpha$ of order $m+1$; and

- The terms $\hat{\alpha}^{m+1} / \mathcal{J}^{m}$ are composed of tree diagrams only, and can be read off from the (negative of the) saddle point of the classical Wilsonian action including both sources and super-wZ term.

Therefore we can write the $\mathcal{O}\left(n^{-m}\right)$ term in $q_{n}$ as

$$
\left.q_{n}\right|_{\mathcal{O}\left(n^{-m}\right)}=\frac{K_{m}}{n^{m}}
$$

where

$$
K_{m}=K_{m}(\alpha)=P_{m+1}(\alpha),
$$

is a polynomial of order $m+1$ in $\alpha$, with the leading term determined by the action of the classical saddle point with super-wZ term included.

It is convenient to eliminate the explicit dependence on $\Delta_{\mathcal{O}}$ and rewrite $q_{n}$ in terms of a series expansion in the charge $\mathcal{J}$ with coefficients $\hat{K}_{m}(\alpha)$ defined by

$$
\left.q_{n}\right|_{\mathcal{O}\left(n^{-m}\right)}=\frac{K_{m}(\alpha)}{n^{m}}=\frac{\Delta_{\mathcal{O}}^{m} K_{m}(\alpha)}{\mathcal{J}^{m}}=\frac{\hat{K}_{m}(\alpha)}{\mathcal{J}^{m}}=\frac{\hat{P}_{m+1}(\alpha)}{\mathcal{J}^{m}}
$$

where the polynomials $\hat{P}_{m+1}(\alpha)$ are simply

$$
\hat{P}_{m+1}(\alpha) \equiv \Delta_{\mathcal{O}}^{m} P_{m+1}(\alpha) .
$$




\subsection{Examples of diagrams}

The nature of the one-point vertices depends how the field $\phi$ is broken up into "background classical solution" and "fluctuation". The simplest starting point is to break up $\phi$ into classical solution and fluctuation, where the classical solution is the solution at $\alpha=0$, with the WZ term ignored. This solution was written down in [1] and on $\mathbb{R}^{4}$ takes the form

$$
\begin{aligned}
\phi_{\mathrm{cl}}(x) & =\frac{e^{i \beta_{0}}\left|x_{1}-x_{2}\right|}{2 \pi\left(x-x_{2}\right)^{2}} \mathcal{J}^{1 / 2}, \quad \bar{\phi}_{\mathrm{cl}}(x)=\frac{e^{-i \beta_{0}}\left|x_{1}-x_{2}\right|}{2 \pi\left(x-x_{1}\right)^{2}} \mathcal{J}^{1 / 2}, \\
\left|\phi_{\mathrm{cl}}(x)\right| & =\frac{\left|x_{1}-x_{2}\right|}{\left|x-x_{1}\right|\left|x-x_{2}\right|} \frac{\mathcal{J}^{1 / 2}}{2 \pi} .
\end{aligned}
$$

Note that these expressions depend on the conformal frame. In the conformal frame of the cylinder they become

$$
\begin{aligned}
\phi_{\mathrm{cl}}(x) & =\frac{e^{t / r}}{2 \pi r} \mathcal{J}^{1 / 2}, \quad \bar{\phi}_{\mathrm{cl}}(x)=\frac{e^{-t / r}}{2 \pi r} \mathcal{J}^{1 / 2}, \\
\left|\phi_{\mathrm{cl}}(x)\right| & =\frac{1}{2 \pi r} \mathcal{J}^{1 / 2} .
\end{aligned}
$$

The dilaton $\tau$ is just constant and the axion $\beta$ is linear in time,

$$
\begin{aligned}
\tau_{\text {cl }}^{\text {[cylinder }]} & =\log (2 \pi \mu R)-\frac{1}{2} \log (\mathcal{J}) \\
\beta_{\mathrm{cl}}^{\text {cylinder }]} & =i \frac{t}{R},
\end{aligned}
$$

which are always properties of a helical classical solution.

Since the $\phi_{\text {classical }}$ solves the classical equation of motion (EOM) exactly at $\alpha=0$, there are no "external" lines without $\alpha$-vertices. With this organization of $\phi$ into background plus fluctuation, there do indeed exist one-point vertices, but each is proportional to $\hat{\alpha}$ and therefore comes with an extra power of $\mathcal{J}^{-1}$. So nontrivial tree diagrams do exist in this organization of diagrams, with each one-point vertex carrying an $\alpha$-factor, which suppresses the weight of the diagram by $\mathcal{J}^{-1}$.

Let us examine the diagramatics of the first few terms in the $1 / n$ expansion of $q_{n}$.

Order $\boldsymbol{n}^{0}$. At order $n^{0}$ we have only the determinant, with no $\alpha$-vertices at all; that is, just the fluctuation determinant of the free vector multiplet action with logarithmic sources proportional to $\mathcal{J}=n \Delta_{\mathcal{O}}$. The sources make this determinant nontrivial, but its value is already known directly by expanding the free-field partition function

$$
q_{n}^{(\text {free })} \equiv \log \left(Z_{n}^{(\text {free })}\right)=\mathbf{A} n+\mathbf{B}+\log \left(\Gamma\left(\Delta_{\mathcal{O}} n+1\right)\right)=\log (\Gamma(\mathcal{J}+1))
$$

to order $n^{0}$. Indeed, all diagrams without $\alpha$-vertices, are simply terms in the expansion of $\log \left(\Gamma\left(\Delta_{\mathcal{O}} n+1\right)\right)=\log (\Gamma(\mathcal{J}+1))$ : the order $\alpha^{0} n^{-m}$ term in $q_{n}$, is just the $n^{-m}$ term in the Stirling series of $\log (\Gamma(\mathcal{J}+1))$ :

$$
\left.q_{n}\right|_{\mathcal{O}\left(\alpha^{0} n^{-m}\right)}=\left.\log (\Gamma(\mathcal{J}+1))\right|_{\mathcal{O}\left(\mathcal{J}^{-m}\right)}=\frac{(-1)^{m} B_{m+1}}{m(m+1)}
$$

where $B_{m+1}$ is the Bernoulli number. 


\begin{tabular}{|ll|}
\hline description & term \\
Two-loop with no $\alpha$-vertices & $\hat{K}_{1,0}$ \\
One-loop with one $\alpha$-vertex & $\hat{K}_{1,1} \alpha$ \\
Tree-level with two $\alpha$-vertices & $\hat{K}_{1,2} \alpha^{2}$
\end{tabular}

Table 1. Diagrams appearing at order $1 / \mathcal{J}$.

At order $n^{0}$ there is also a "diagram" with one $\alpha$-vertex that has no external lines at all: this is just the evaluation of the (negative of the Euclidean) WZ term on the classical solution, whose $\log (n)$ piece was computed in [1]. In that paper we did not compute the non-logarithmic contribution to $q_{n}$ at order $n^{0}$. Indeed this term by itself is ill-defined due to the conformal anomaly; only the difference $q_{n}-q_{0}$ is well-defined. While the $n^{0}$ term in $q_{n}-q_{0}$ is well-defined and in principle computable, the computation requires a somewhat careful matching of conventions and renormalization schemes between the sphere partition function and the large- $\mathcal{J}$ partition function, and we do not pursue it in the present paper. We focus instead on large- $n$ limits of differences $q_{n+1}-q_{n}$ between adjacent correlators, which are also well-defined and amenable to direct analysis at large $n$.

From equation (2.18) we see that there are no further contributions at order $n^{0}$.

Order $\boldsymbol{n}^{-1}$. Consulting equation (2.18), we see that the order $n^{-1}$ contribution to $q_{n}$ contains three distinct types of diagram (see table 1):

- Two-loop diagrams with no $\alpha$-vertices;

- One-loop diagrams with one $\alpha$-vertex; and

- Tree-level diagrams with two $\alpha$-vertices.

We shall call these respective contributions

$$
\left.q_{n}\right|_{O\left(n^{-1}\right)} \equiv \frac{\hat{K}_{1}}{\mathcal{J}} \equiv \frac{\hat{P}_{2}(\alpha)}{\mathcal{J}} \equiv \sum_{a=0}^{2} \frac{\hat{K}_{1, a} \alpha^{a}}{\mathcal{J}}
$$

- The two-loop diagram would be cumbersome to compute directly, but we do not have to: we know it is equal to the $\mathcal{J}^{-1}$ term in the expansion of $\log (\Gamma(\mathcal{J}+1))$. So from eq. (2.28) we have:

$$
\hat{K}_{1,0}=\frac{B_{2}}{2}=+\frac{1}{12} .
$$


- We calculate $\hat{K}_{1,2}$ in section $\mathrm{E}$ of the appendix, and the result is in eq. (E.31) (for $m=1)$ :

$$
K_{1,2} \equiv \Delta_{\mathcal{O}}^{-1} \hat{K}_{1,2}=+\frac{1}{4} \text { for the case } \Delta_{\mathcal{O}}=2,
$$

which according to (2.21) means

$$
\hat{K}_{1,2}=2 \times K_{1,2}=+\frac{1}{2} .
$$

- The value $\hat{K}_{1,1}$ comes from of the one-loop diagram with a single $\alpha$-vertex. This diagram is also somewhat tedious to compute directly and we will not need to do so. Instead, we will infer the value of $\hat{K}_{1,1}$ based on the known values of $\hat{K}_{1,0}$ and $\hat{K}_{1,2}$ together with the value of $\hat{K}_{2}$ for $\alpha=1$, which is realized by $\mathcal{N}=4$ SYM and has a simple closed-form expression for $q_{n}$.

$$
q_{n}^{\mathcal{N}}=4 \mathrm{SYM}=\mathbf{A} n+\mathbf{B}+\log (\Gamma(2 n+2)) .
$$

This theory has $\Delta_{\mathcal{O}}=2$ and $\alpha=1$ (see [1] for the normalization of the $\alpha$-coefficient) and so using Stirling's formula we have

$$
\left.q_{n}\right|_{\mathcal{O}(1 / n)}=K_{1}^{(\mathcal{N}=4 \mathrm{SYM})}=\frac{13}{24} .
$$

Then, using the definition of $P_{2}$ we have

$$
\begin{aligned}
\frac{13}{24} & =K_{1}^{(\mathcal{N}=4 \text { SYM })}=\left.K_{1}\right|_{\Delta_{\mathcal{O}}=2, \alpha=1}=\left.P_{2}(\alpha=1)\right|_{\Delta_{\mathcal{O}}=2}=\frac{1}{2} \hat{P}_{2}(\alpha=1)= \\
& =\frac{1}{2}\left(\hat{K}_{1,0}+\hat{K}_{1,1}+\hat{K}_{1,2}\right)=\frac{7}{24}+\frac{1}{2} \hat{K}_{1,1} .
\end{aligned}
$$

This gives

$$
\hat{K}_{1,1}=+\frac{1}{2} .
$$

The final result is that the general form of the coefficient of the $1 / \mathcal{J}$ term is

$$
\hat{K}_{1}=\hat{P}_{2}(\alpha)=\frac{\alpha^{2}}{2}+\frac{\alpha}{2}+\frac{1}{12}=\frac{1}{2}\left[\alpha^{2}+\alpha+\frac{1}{6}\right] .
$$

Later we will be interested in the case of $\mathcal{N}=2$ SQCD with $N_{f}=4$, which has $\alpha=\frac{3}{2}$ and $\Delta_{\mathcal{O}}=1$. This corresponds to a value of

$$
\begin{array}{r}
\hat{K}_{1}\left(\alpha=\frac{3}{2}\right)=+\frac{47}{24}, \\
\hat{K}_{1}\left(\Delta_{\mathcal{O}}=2, \alpha=\frac{3}{2}\right)=+\frac{47}{48} .
\end{array}
$$

We will be able to check this value against correlation functions computed by recursion relations starting from the sphere partition function $Z=Z_{0}=\exp \left\{q_{0}\right\}$. 


\subsection{Universality for power law corrections versus nonuniversality for expo- nential corrections}

Combining the results from [1] and section 2.3, we find that the correlation functions $Z^{-1} Z_{n}=\exp \left\{q_{n}-q_{0}\right\}$ take the form

$$
q_{n} \simeq \mathcal{J} \log (\mathcal{J})+\left(\alpha+\frac{1}{2}\right) \log (\mathcal{J})+(\mathbf{A}-1) \mathcal{J}+\mathbf{B}+\log \sqrt{2 \pi}+\sum_{m \geq 1} \frac{\hat{K}_{m}(\alpha)}{\mathcal{J}^{m}},
$$

where $\mathcal{J}=n \Delta_{\mathcal{O}}$ and $\hat{K}_{m}=\hat{P}_{m+1}(\alpha)$ are some universal coefficients independent of the microscopic details of the underlying microscopic CFT including the dimension $\Delta_{\mathcal{O}}$ of the generator of the Coulomb branch and depending only on $\alpha$ as a theory-independent polynomial of order $m+1$. The coefficients $\mathbf{A}$ and $\mathbf{B}$ can depend on the theory overall, on the normalization of the operators $\mathcal{O}$, on the marginal parameters $\tau$ if any, and on the renormalization scheme. The coefficients $\hat{K}_{m}$, on the other hand, are independent of the renormalization scheme and marginal couplings, and can be computed in the effective theory with no counterterm ambiguities because no superconformal $F$-terms exist of order $\mathcal{J}^{-1}$ or higher, a property special to theories with one-dimensional Coulomb branches.

The $\simeq$ in the formula indicates that the formula above should be understood only as an asymptotic expansion in $\mathcal{J}$ : the EFT can be valid only up to amplitudes associated with propagation of massive particles over the infrared scale. In a conformal theory, the mass $M$ of the lowest massive excitation must be given by $M=\kappa_{\mathrm{M}}|\phi|$ where $\kappa_{M}$ is a dimensionless parameter depending on the theory overall and on the marginal couplings. In familiar cases we know $\kappa_{\mathrm{M}}$ depends on the gauge coupling, as $\propto g_{\mathrm{YM}}{ }^{+1} \propto \operatorname{Im}(\tau)^{-1 / 2}$ at weak coupling. Thus we expect EFT to break down due to effects of $\operatorname{size} \exp \left(-\kappa_{M} L\right)$ where $L \equiv \kappa_{L} \times r$, with $r$ being the radius of the sphere and $\kappa_{L}$ being a dimensionless number. Then the size of the exponentially small effects signaling the breakdown of the EFT, should go as $\exp \left(-\kappa_{M} \kappa_{L} r|\phi|\right)=\exp (-\kappa \sqrt{\mathcal{J}})$, where

$$
\kappa=2 \pi \kappa_{M} \kappa_{L} .
$$

Here we have used the identity in eq. (2.24):

$$
\mathcal{J}=4 \pi^{2} r^{2}|\phi|^{2}
$$

for the classical helical ground-state solution.

Since $\kappa$ contains a factor of $\kappa_{\mathrm{M}}$, and since $\kappa_{\mathrm{M}}$ is theory dependent (in particular, depending on marginal parameters), we do not expect the theory-independence of the $n^{-m}$ terms to extend to the exponentially small corrections. We shall return to this point later on.

\section{Lagrangian theories}

\subsection{Derivation of the $\mathcal{J}^{-m}$ corrections in Lagrangian theories}

In [15-18, 29], Coulomb branch correlation functions were analyzed for $\mathcal{N}=2$ sCFTs with a marginal coupling $\tau$. In these, the correlation functions were shown to obey recursion 
relations with respect to the coupling constant $\tau$ :

$$
\partial \bar{\partial} q_{n}(\tau, \bar{\tau})=\exp \left[q_{n+1}(\tau, \bar{\tau})-q_{n}(\tau, \bar{\tau})\right]-\exp \left[q_{n}(\tau, \bar{\tau})-q_{n-1}(\tau, \bar{\tau})\right] .
$$

In particular, for $\mathcal{N}=2$ superconformal gauge theories with $\mathrm{SU}(2)$ or $\mathrm{SO}(3)$ gauge group, the recursion relations in eq. (3.1) are sufficient to determine all correlation functions of Coulomb branch chiral primaries for any value of $\alpha$ where the EFT can be completed by a superconformal gauge theory.

In appendix $\mathrm{A}$, we show that the recursion relations, when they apply, fix the $\mathcal{J}^{-m}$ power-law corrections uniquely for a given value of $\alpha$. In the derivation, we use only the fact that $q_{n}$ has the asymptotic expansion (2.39) with $\hat{K}_{m}$ depending only on $\alpha$ and not on $\tau, \bar{\tau}$ or $\Delta_{\mathcal{O}}$, a property which follows from the properties of the Coulomb-branch EFT as discussed above. We see in eq. (A.22) that the recursion relations uniquely fix

$$
q_{n} \simeq \mathcal{J} \mathbf{A}+\mathbf{B}+\log (\Gamma(\mathcal{J}+\alpha+1))
$$

and the coefficients in the perturbative expansion are those in eq. (A.23):

$$
\hat{K}_{m}=\hat{P}_{m+1}^{[\text {univ] }}(\alpha) \equiv \text { coefficient of } \mathcal{J}^{-m} \text { in } \log (\Gamma(\mathcal{J}+\alpha+1)),
$$

for any value of $\alpha$ corresponding to a gauge theory with marginal coupling. These are essentially the Bernoulli polynomials of degree $m+1$ :

$$
\hat{P}_{m+1}^{[\mathrm{univ}]}(\alpha)=\frac{(-)^{m+1}}{m(m+1)} B_{m+1}(\alpha+1) .
$$

For concreteness, we give the first several values of $\hat{P}_{m+1}^{[\text {univ] }}(\alpha)$ :

$$
\begin{aligned}
& \hat{P}_{2}^{\text {[univ] }}(\alpha)=\frac{1}{2} \alpha^{2}+\frac{1}{2} \alpha+\frac{1}{12}, \\
& \hat{P}_{3}^{\text {[univ] }}(\alpha)=-\frac{1}{6} \alpha^{3}-\frac{1}{4} \alpha^{2}-\frac{1}{12} \alpha, \\
& \hat{P}_{4}^{\text {[univ] }}(\alpha)=+\frac{1}{12} \alpha^{4}+\frac{1}{6} \alpha^{3}+\frac{1}{12} \alpha^{2}-\frac{1}{360} \\
& \hat{P}_{5}^{\text {[univ] }}(\alpha)=-\frac{1}{20} \alpha^{5}-\frac{1}{8} \alpha^{4}-\frac{1}{12} \alpha^{3}+\frac{1}{120} \alpha, \\
& \hat{P}_{6}^{\text {[univ] }}(\alpha)=+\frac{1}{30} \alpha^{6}+\frac{1}{10} \alpha^{5}+\frac{1}{12} \alpha^{4}-\frac{1}{60} \alpha^{2}+\frac{1}{1260}, \\
& \hat{P}_{7}^{[\text {univ] }}(\alpha)=-\frac{1}{42} \alpha^{7}-\frac{1}{12} \alpha^{6}-\frac{1}{12} \alpha^{5}+\frac{1}{36} \alpha^{3}-\frac{1}{252} \alpha,
\end{aligned}
$$

and so forth. The coefficient of $\alpha^{m+1}$ in $\hat{P}_{m+1}$ is $(-1)^{m+1} /(m(m+1))$. We show in appendix E that these values can be independently computed in our EFT without using to the recursion relations. On the other hand, these polynomials satisfy an effective recursion relation

$$
\frac{\mathrm{d} \hat{P}_{m+1}^{[\text {univ] }}(\alpha)}{\mathrm{d} \alpha}=-(m-1) \hat{P}_{m}^{[\text {univ }]}(\alpha)
$$

which is independent of any notion of coupling. 
Note that we do not use any information about the sphere partition function in our derivation. As emphasized in [15], the solution to the recursion relations is not unique, and one needs "boundary conditions" of some kind to select the correct solution. For the case of rank-one superconformal gauge theories, the sphere partition function uniquely determines all the $q_{n}$. Here we show that, without using the sphere partition function as an input, the large $-n$ asymptotics corresponding to quantization of the Coulomb-branch EFT, fix the correlators not completely uniquely, but uniquely up to corrections smaller than any power of $n$. In the next section, we shall present evidence that the asymptotic expansion in inverse powers of $\mathcal{J}$ produces the physically correct answer, matching the correlators extracted from the sphere partition function, to exponentially fine accuracy as a function of $\mathcal{J}$. We shall also return later to discuss the physical meaning of these exponentially small corrections.

\subsection{Examples}

In this section we consider examples of gauge theories with marginal couplings and the power-law terms $K_{m} n^{-m}=2^{-m} \hat{K}_{m} n^{-m}=\hat{K}_{m} \mathcal{J}^{-m}$ in their large- $\mathcal{J}$ expansions.

Abelian gauge theory without matter: $\boldsymbol{\alpha}=\mathbf{0}$. The Abelian gauge theory with no matter is a gauge theory with a marginal coupling $\tau$. This coupling is a true parameter of the theory, affecting for instance the spectrum of electric and magnetic flux states on a spatial slice $\Sigma$ with a nonvanishing second homology group; the Abelian gauge theory is therefore a gauge theory with a marginal coupling, which must obey the recursion relation in eq. (3.1) and therefore have $\hat{K}_{m}=\hat{P}_{m+1}$.

The Coulomb branch chiral ring is generated by $\phi$, so $\mathcal{O}=\phi$ and $\Delta_{\mathcal{O}}=1$. The twopoint functions $\mathcal{Y}_{n}$ are particularly easy to compute in this case because the flux states are irrelevant to the computation of correlation functions of local operators. Therefore $\tau$ decouples completely from such correlators, except through the normalization of the vector multiplet scalar $\phi_{\text {holo }}$, which drops out if we consider correlators of $\phi \equiv \phi_{\text {unit }}$.

The correlation function of $\phi^{n}$ with $\bar{\phi}^{n}$ is thus given by

$$
\mathcal{Y}_{n}=\mathbf{N}_{\mathcal{O}}^{n} \Gamma(n+1)=\mathbf{N}_{\mathcal{O}}^{n} \Gamma(n+1)
$$

and so we have

$$
K_{m}=P_{m+1}^{[\text {univ }]}=\text { coefficient of } \mathcal{J}^{-m} \text { in } \log (\Gamma(\mathcal{J}+1)),
$$

in agreement with formula in eq. (3.3).

$\mathcal{N}=4$ super-Yang-Mills with $g=s u(2): \alpha=1$. Now we consider the case of $\mathcal{N}=4$ sYM with $g=s u(2)$. The generator of the chiral ring is $\mathcal{O} \propto \operatorname{Tr}\left(\hat{A}^{2}\right)$, where $\hat{A}$ is the adjoint-valued vector-multiplet scalar in the microscopic theory, giving $\mathcal{O}$ dimension $\Delta_{\mathcal{O}}=2$, as in all rank-one gauge theories. The correlation functions in this case are

$$
\exp \left\{q_{n}\right\}=Z \mathcal{Y}_{n}=\mathbf{N}^{n} \log [(2 n+1) !]=\log (\Gamma(\mathcal{J}+2)),
$$


and so the power-law corrections are

$$
\begin{aligned}
\hat{K}_{m} & =\text { coefficient of } \mathcal{J}^{-m} \text { in } \log (\Gamma(\mathcal{J}+2))=(-)^{m+1}\left(\frac{1}{m}+\frac{B_{m+1}}{m(m+1)}\right) \\
& =\frac{(-)^{m+1}}{m(m+1)} B_{m+1}(2),
\end{aligned}
$$

where $B_{m+1}(x)$ is the Bernoulli polynomial. This agrees with formula in eq. (3.3) for the case $\alpha=\alpha_{\mathcal{N}=4, \mathrm{G}=\mathrm{SU}(2)}=1$.

$\mathcal{N}=2$ super-QCD with $g=s u(2)$ and $N_{F}=4: \alpha=3 / 2$. Now we consider the more involved case of conformal $\mathcal{N}=2 \mathrm{SQCD}$ with $g=s u(2)$ and four hypermultiplets in the 2 representation of $\mathrm{SU}(2)$. Here, the coupling constant dependence is complicated even for the sphere partition function without insertions; the correlation functions $\mathcal{Y}_{n}(\tau, \bar{\tau})$ which are obtained from $Z(\tau, \bar{\tau})$ by the recursion relations, are more complicated still and the complication grows quickly with $n$.

At large $n$, on the other hand, formula in eq. (A.22) tells us the dependence of $\mathcal{Y}_{n}$ on the coupling is just the trivial $[\operatorname{Im}(\tau)]^{-2 n}$ geometric dependence, up to exponentially small corrections to $\log \left(\mathcal{Y}_{n}\right)$. In particular, the corrections should obey the universal formula for power law corrections for rank-one Coulomb-branch correlators, with $\Delta_{\mathcal{O}}=2$ and $\alpha=\alpha_{\mathrm{SQCD}}=3 / 2$ :

$$
\begin{aligned}
K_{m} & =\left.2^{-m} \hat{K}_{m}\right|_{\alpha=\frac{3}{2}}=2^{-m} \hat{P}_{m+1}(\alpha)= \\
& =\text { coefficient of } \mathcal{J}^{-m} \text { in } \log (\Gamma(\mathcal{J}+5 / 2))=\frac{(-1)^{m}}{m(m+1)} B_{m+1}(5 / 2) .
\end{aligned}
$$

Lacking a closed-form expression, we instead compare our prediction (3.16) with data from the numerical evaluation of correlation functions, as in [1].

We begin with the zero-instanton approximation to the sphere partition function $Z=$ $Z_{0}=\exp \left\{q_{0}\right\} \rightarrow Z^{\text {[zero-instanton] }}$ and evolve up to $n=40$ using the recursion relations to get an approximate answer; we expect that the omission of instanton effects should lead to errors no larger than relative size $\exp \{-2 \pi \operatorname{Im}(\tau)\}$, which is smaller than $2 \times 10^{-3}$ for $\operatorname{Im}(\tau)>1$.

In the appendix $\mathrm{F}$ we present data for correlation functions. We take the second difference with respect to $n$ in $q_{n}$ to cancel the $n^{0}$ and $n^{1}$ terms in $\mathcal{Y}_{n}$,

$$
\triangle_{n}^{2} q_{n}=q_{n+2}-2 q_{n+1}+q_{n},
$$

and we compare the $m^{-1}$ and smaller terms.

We find that the numerical values are in beautiful agreement with the prediction (3.16). Quite rapidly, already for $\tau \simeq 4 i$, the $\tau$-dependence drops for all values of $n$. The asymptotic values is well approximated by our prediction $\triangle_{n}^{2} q_{n}^{\mathrm{EFT}}$ for $n$ larger that $n \gtrsim$ 5 , where the discrepancy between the EFT result and the localization is of order 1 $\triangle_{n}^{2} q^{\mathrm{EFT}} /\left.\triangle_{n}^{2} q^{(\mathrm{loc})}\right|_{n=5, \operatorname{Im}(\tau) \lesssim 1} \approx 1 \%$. Even for $n=1$, the discrepancy is only of order $1-\triangle_{n}^{2} q^{\mathrm{EFT}} /\left.\triangle_{n}^{2} q^{(\mathrm{loc})}\right|_{n=1, \operatorname{Im}(\tau) \leqslant 1} \approx 8 \%$ (see figure 1). In section 5, we will estimate the behavior of the discrepancy as function of $\mathcal{J}$ and $\operatorname{Im} \tau$. 


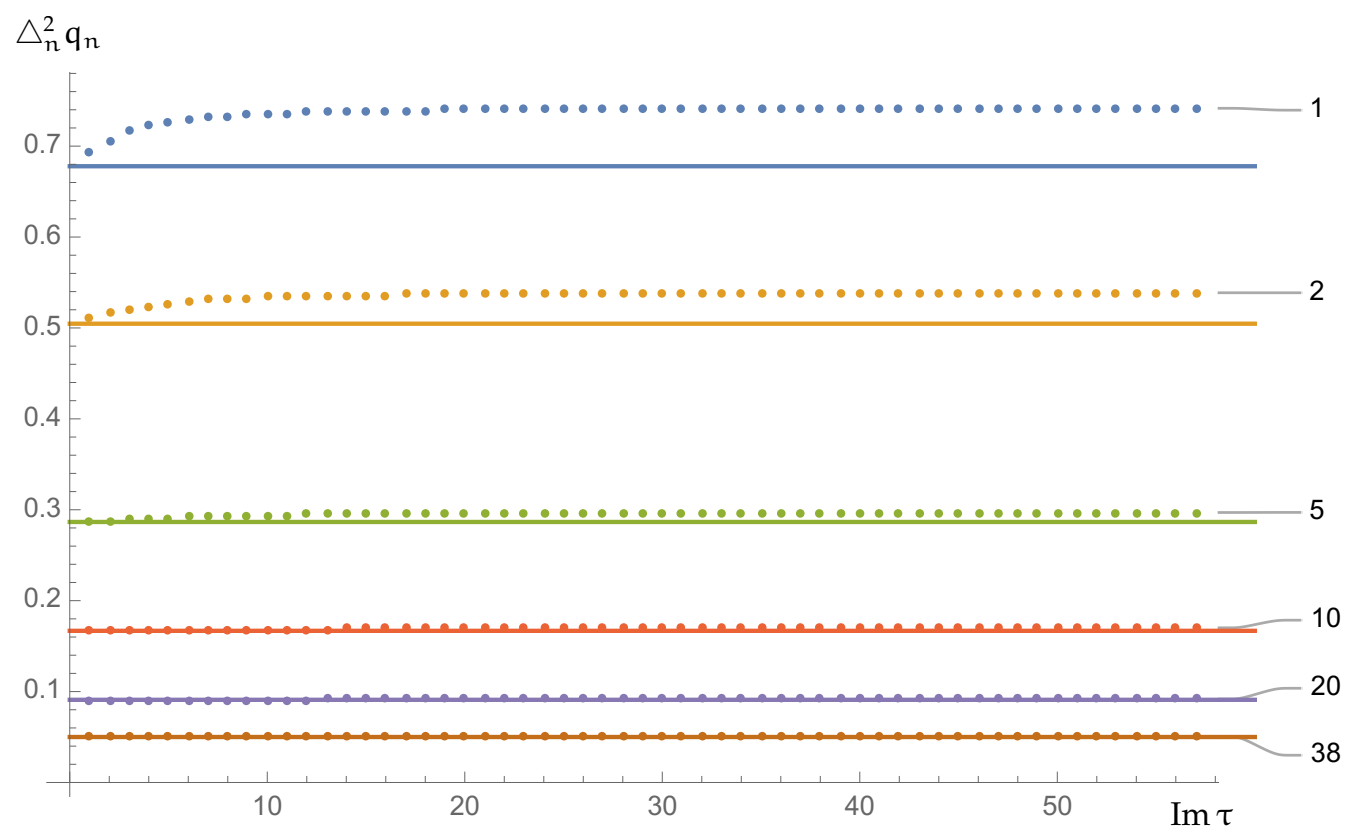

Figure 1. Second difference in $n$ for $\triangle_{n}^{2} q_{n}^{(\text {loc })}$ (dots) and for $\triangle_{n}^{2} q_{n}^{\mathrm{EFT}}$ (continuous lines) as function of $\operatorname{Im} \tau$ at fixed values of $n$. The numerical results quickly reach a $\tau$-independent value that is well approximated by the asymptotic formula when $n$ is larger than $n \gtrsim 5$.

\section{Universal theory-independence of the $\mathcal{J}^{-m}$ corrections}

\subsection{Initial comments}

The derivation of the $1 / n$ corrections in section 3 , has relied beyond first order on the recursion relations given in eq. (3.1). These recursion relations, as derived in [29], apply only to theories with a marginal coupling. Despite this, the actual formula for the powerlaw corrections is completely independent of the marginal coupling, depending only on the $\alpha$-coefficient of the theory. It is tempting, therefore, to wonder whether the formula may also apply to rank-one theories with other values of $\alpha$ (for instance those in the classification of Argyres et al., [30-33]), most of which do not have a marginal coupling at all. In this section we shall present arguments suggesting that the formula in eq. (3.2) for the power-law corrections should apply to any value of $\alpha$, for Coulomb-branch chiral-primary correlators in any rank-one theory with any value of $\alpha$, whether or not it has a marginal coupling.

For infinitely many values of $\alpha$, the EFT has a nonunitary but superconformally invariant regulator obtained by adding adjoint and fundamental hypermultiplets, of which either or both have the opposite statistics to that of a unitary matter field. These nonunitary theories do have marginal couplings, and we observe that the derivation of the recursion relations works equally well as in the unitary case since this derivation does not rely on unitarity at all.

\subsection{Ultraviolet regulators with marginal couplings}

For three values of $\alpha$, namely $\alpha=0,+1,+\frac{3}{2}$, the Coulomb-branch EFT has a unitary UV completion which in the first case is exactly free and in the latter two cases has a marginal 
coupling parameter. These are the only three unitary SCFTs with marginal coupling and one-dimensional Coulomb branch.

We note, however, that unitarity ${ }^{2}$ appears to play no role in the derivation of the recursion relations. We can therefore consider gauge theories with both nonunitary as well as unitary matter sectors. If the matter is chosen so that the $\beta$-function vanishes identically, these gauge theories will have marginal gauge coupling $\tau$ and values of the $\alpha$-coefficient $\alpha=\frac{5}{12} \frac{a_{\mathrm{CFT}}-a_{\mathrm{EFT}}}{a_{\text {free } \mathrm{U}(1) \text { vector }}}$.

The theories are constructed by adding "ghost" hypermultiplets — with the same super-conformal transformations and R-symmetry quantum numbers as ordinary hypermultiplets, but with spin-statistics reversed - that is, a multiplet whose lowest component is a scalar fermion, transforming in the $\mathbf{2}$ of the $\mathrm{SU}(2) \mathrm{R}$-symmetry group.

We give some details of these theories in appendix D. The relevant facts are the $\beta$-function cancellation condition (D.6) and the anomaly mismatch $\alpha$-coefficient of the Coulomb branch EFT. If there are $n_{\mathbf{R}}^{\text {(hyper) }}$ in a representation $\mathbf{R}$ of the gauge group, and some number of ghost hypers $n_{\mathbf{R}}^{\text {(ghost hyper) }}$ in a representation of the gauge group, then the hypers contribute to the $\beta$-function and Weyl anomaly through the difference between the two,

$$
\tilde{n}_{\mathbf{R}}^{\text {(hyper) }} \equiv n_{\mathbf{R}}^{\text {(hyper) }}-n_{\mathbf{R}}^{\text {(ghost hyper) }} .
$$

We can construct many interesting nonunitary SCFTs this way, but the simplest choice is to take only hypers and ghost hypers in the $\mathbf{2}$ and $\mathbf{4}$ representations, in which case the $\beta$-function vanishes if

$$
\tilde{n}_{2}^{\text {(hyper) }}=8-10 \tilde{n}_{4}^{\text {(hyper) }},
$$

and the theory is conformal. There are no massless degrees of freedom on the Coulomb branch other than the massless $\mathrm{U}(1)$ vector multiplet, and the value of the $\alpha$-coefficient is

$$
\alpha=\frac{3}{2}-\frac{4}{3} \tilde{n}_{4} .
$$

This shows that the Coulomb-branch EFT with super-WZ $\alpha$-coefficient $\alpha=\frac{3}{2}-\frac{4}{3} \tilde{n}_{4}$ can be obtained with a manifestly $\mathcal{N}=2$ superconformal regulator, analogous to a Pauli-Villars regulator.

Other values of $\alpha$ are obtainable using $\mathcal{N}=2$ superconformal gauge theories with ordinary and ghost hypermultiplets in higher representations of $\mathrm{SU}(2)$, but for the present purposes it suffices to show how an infinite number of values of $\alpha$ may be obtained with such constructions.

Apart from the manifest superconformal invariance, a second valuable feature of this regulator is the fact that it possesses a marginal coupling and thus obeys the recursion relations of [29] when considered as a full SCFT rather than as a regulator for the vector multiplet EFT. Though the recursion relations were originally applied in the context of unitary superconformal gauge theory, unitarity of the CFT appears to play no essential role in the derivation of the relations, and thus one expects $\mathcal{N}=2$ superconformal gauge theories with ghost-hypers to obey the same recursion relations as those with ordinary

\footnotetext{
${ }^{2}$ Or more precisely, reflection positivity in the Euclidean path integral.
} 
matter. We will now make use of this fact to write recursion relations for the power-law corrections for more general values of the $\alpha$-coefficient.

\subsection{Universal polynomials}

In the previous section, we have observed that there appear to be an infinite number of rank-one $\mathcal{N}=2$ superconformal gauge theories, realized by $\mathrm{SU}(2)$ gauge theory with combinations of ordinary hypermultiplets with ghost hypermultiplets. By taking such combinations, we find we can regulate any one-dimensional Coulomb-branch EFT with $\alpha$ coefficient satisfying $\alpha \in \frac{3}{2}-\frac{4}{3} \mathbb{Z}$ by a superconformal $\mathrm{SU}(2)$ gauge theory with ordinary hypers and ghost hypers in the 2- and 4-dimensional representations. These theories do have the marginal coupling constant $\tau$ and must therefore obey the recursion relations with respect to $\tau$, as in [29].

As we have shown diagrammatically in section 2.3 , the $1 / n^{m}$ corrections to the logarithms of the two-point functions can depend only on $\mathcal{J}$ and $\alpha$, and not on $\Delta_{\mathcal{O}}$ and $n$ individually, nor on marginal couplings, nor on any other details of the microscopic completion, whether it be a unitary quantum field theory or an artificial regulator, so long as the regulator preserves exact $\mathcal{N}=2$ superconformal symmetry and possesses an exact conformal manifold parametrized by the gauge coupling $\tau$. This means the terms $K_{m}=P_{m}(\alpha)$ are universal polynomials, common to any superconformal quantization of the EFT, whether Lagrangian or non-Lagrangian, unitary or nonunitary. At the same time, the recursion relations establish that

$$
\hat{P}_{m+1}(\alpha)=\hat{P}_{m+1}^{[\text {univ }]}(\alpha)
$$

for any superconformal UV-completion obeying the recursion relations, with $\hat{P}_{m+1}^{[\text {univ] }}(\alpha)$ defined in eq. (3.4), whether the completion is unitary or not. Since there appear to exist nonunitary superconformal regulators for the Coulomb-branch EFT for an infinite number of distinct values of $\alpha$, it follows that $\hat{P}_{m+1}(\alpha)=\hat{P}_{m+1}^{\text {[univ] }}(\alpha)$ must agree for an infinite number of values, for any $m \geq 1$. Any two polynomials of order $m+1$ that agree for $m+1$ or more values, must agree identically, and we conclude

$$
\hat{P}_{m+1}(\alpha)=\hat{P}_{m+1}^{[\text {univ] }}(\alpha)=\text { coefficient of } \mathcal{J}^{-m} \text { in } \log (\Gamma(\mathcal{J}+\alpha+1))
$$

for all values of $\alpha$.

Assuming the accuracy of our inferences about the properties of the ghost-hyper regulators, this establishes the formula for $\hat{P}_{m+1}(\alpha)$ for any value of $\alpha$, independent of any reference to a UV-completion. In particular, the polynomials $\hat{P}_{m+1}(\alpha)$ must also give the power-law corrections $\hat{K}_{m} / \mathcal{J}^{m}$ for correlators of Coulomb-branch chiral primaries in the non-Lagrangian theories in [30-35].

\section{Universal EFT behavior compared with $S^{4}$ localization}

In section 2.5, we showed that the EFT approximation to the $q_{n}$ must be universal to all orders in inverse powers of $\mathcal{J}=n \Delta_{\mathcal{O}}$, but not universal nonperturbatively in $n$. In 
this section, we check this claim in detail using the $q_{n}$ correlation functions in $\mathcal{N}=2$ superconformal SQCD with $G=\mathrm{SU}(2)$ and $N_{F}=4$, as computed from the $S^{4}$ partition function as done in [15-18, 29].

This method makes use of the BPS property of the two-point function, using recursion relations to obtain a closed-form expression for each $q_{n+2}(\tau, \bar{\tau})$ in terms of the two previous amplitudes $q_{n+1}(\tau, \bar{\tau})$ and $q_{n}(\tau, \bar{\tau})$, for any rank-one theory with a marginal coupling. Since $q_{0}(\tau, \bar{\tau})$ can be computed unambiguously (up to holomorphic scheme-dependence [36] which correspond to Kahler transformations of the conformal-manifold Kahler potential and which cancel out in the normalized correlation functions) by supersymmetric localization, this gives in principle a closed form expression for every $q_{n}$. In practice, even the sphere partition function $q_{0}$ itself is quite a complicated function of $\tau$ and $\bar{\tau}$, and its evolution to higher $q_{n}$ by the recursion relations grows rapidly in complexity with higher $n$, making exact evaluation of large-order $q_{n}$ intractable for $n$ moderately large.

We can evade this difficulty by taking only the perturbative piece of the sphere partition function as an initial condition, and considering only the second difference in eq. (3.17) $\triangle_{n}^{2} q_{n}=q_{n+2}-2 q_{n+1}+q_{n}$, as in [1]. This has two advantages. First, the difference $\triangle_{n}^{2} q_{n}$ removes the theory-dependent coefficients $\mathbf{A}$ and $\mathbf{B}$ of the $\mathcal{J}^{1}$ and $\mathcal{J}^{0}$ terms from the asymptotic expansion, and allows us to isolate the power corrections and exponentially small terms. Explicitly

$$
\triangle_{n}^{2} q_{n}^{\mathrm{EFT}}=\log \left(\frac{(2 n+\alpha+3)(2 n+\alpha+4)}{(2 n+\alpha+1)(2 n+\alpha+2)}\right)
$$

Second, we make use of the fact that our predicted values for the power-corrections are universal, and therefore coupling-independent. We can therefore take a limit in which gauge instantons are unimportant. In particular, we can take a weak-coupling limit $\tau \rightarrow+i \infty$.

We must be somewhat careful to take the limit in such a way that the nonuniversal exponentially small corrections are not enhanced by taking weak coupling: the validity of the EFT depends on the ratio between the infrared scale and the physical masses of the lowest massive excitations, such as the hypermultiplets and the $W$-bosons. If we set this ratio too small, the validity of the EFT would break down entirely, and in particular we would expect the exponentially small corrections $\exp \left\{-M / E_{\mathrm{IR}}\right\}$ associated with macroscopic virtual propagation of massive particles, to become large if we were to take $\tau \rightarrow+i \infty$ at fixed $\mathcal{J}$. The clash of limits between $\tau \rightarrow i \infty$ at fixed $\mathcal{J}$, and $\mathcal{J} \rightarrow \infty$ at fixed $\tau$ is inevitable for a simple reason: the Coulomb-branch EFT is obtained by integrating out the massive excitations, the $W$-bosons and hypermultiplets. At fixed $\left|\phi_{\text {unit }}\right| \propto \sqrt{\mathcal{J}} / r$ the masses of the lightest massive excitations go as $g_{\mathrm{YM}}\left|\phi_{\text {unit }}\right|$. If we fix $\mathcal{J}$, at however large a value, taking the weak-coupling limit always brings massive excitations to masses below our Wilsonian cutoff $\Lambda$, and the predictions of the EFT are no longer valid. If on the other hand we fix $\operatorname{Im} \tau$ at however large a value, and take $\mathcal{J} \rightarrow \infty$, the weak coupling predictions are invalidated by the combinatorics of the diagrams: for sufficiently many external legs, loop corrections to correlators are enhanced by powers of $\mathcal{J}$ and gauge-theoretic perturbation theory breaks down at arbitrarily weak coupling $\mathcal{J}^{-1 / 2} \lesssim g_{\mathrm{YM}} \ll 1$. 


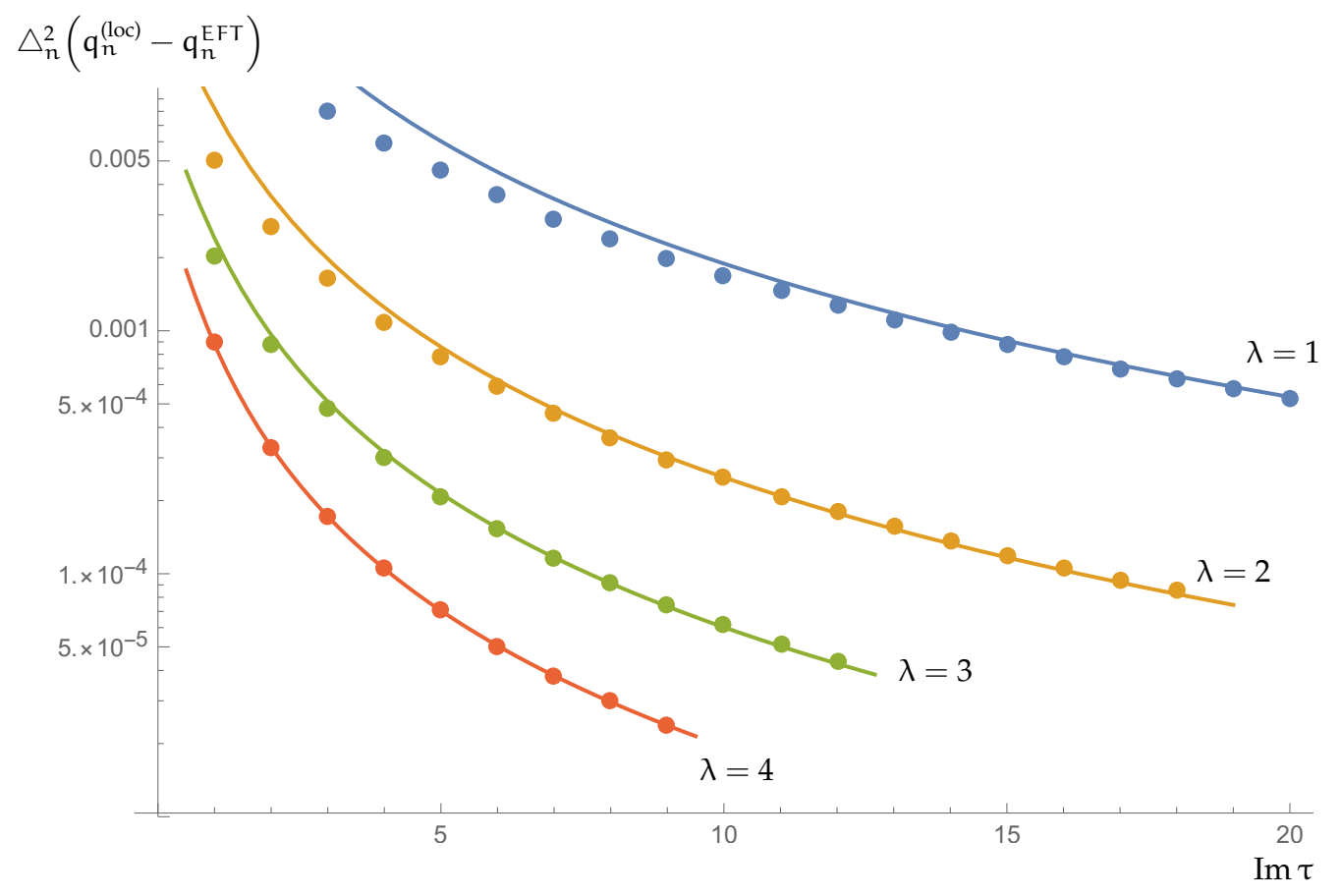

Figure 2. Second difference in $n$ for the discrepancy between localization and EFT results $\triangle_{n}^{2}\left(q_{n}^{\text {(loc) }}-q_{n}^{\mathrm{EFT}}\right)$ (dots) compared to $\triangle_{n}^{2}\left(A_{1} e^{-A_{2} \sqrt{n / \operatorname{Im} \tau}}\right)$ (continuous lines) as functions of $\operatorname{Im} \tau$ at fixed values of $n / \operatorname{Im} \tau$ at $A_{1}=1.8, A_{2}=3.2$. The agreement is quite good already for $\lambda=3$.

The solution to this problem is to take the double-scaling limit of [2], in which $\mathcal{J}$ is taken to infinity with $\lambda=2 \pi \mathcal{J} / \operatorname{Im} \tau$ held fixed. The ratio of the infrared scale to the mass of the heavy excitations is fixed in terms of $\lambda$, so in this limit gauge instantons are suppressed while virtual macroscopic massive propagation is suppressed exponentially as $\exp \left(-\right.$ const. $\left.\times \lambda^{1 / 2}\right)$.

In appendix F, we compare the sum rule for the universal power law formula with the data of the sphere partition function in the double scaling limit, in the case of $\mathcal{N}=2 \mathrm{SQCD}$ with $G=\mathrm{SU}(2)$ and $N_{f}=4$. We find a remarkably accurate agreement and then can estimate the leading correction to be

$$
\left.\log \left(\mathcal{Y}_{n}\right)\right|_{\text {localization }} ^{\mathrm{SQCD}}-\left.\log \left(\mathcal{Y}_{n}\right)\right|_{\mathrm{EFT}} ^{\mathrm{SQCD}} \approx A_{1} e^{-A_{2} \sqrt{\mathcal{J} /(2 \operatorname{Im} \tau)}}
$$

where $A_{1}=1.8(2)$ and $A_{2}=3.2(1)$. In figure 2 we show how this simple form already reproduces the localization data for values of $\lambda \approx 3$. In figure 3 we show how adding this contribution improves the agreement between our prediction and the localization data also at smaller values of $n$ (this is to be compared with the purely perturbative results shown in figure 1).

Approximation of the difference equation by a differential equation. For sufficiently large $n$, we can approximate the $n$-dependent linear difference equation for $\delta$ by an 


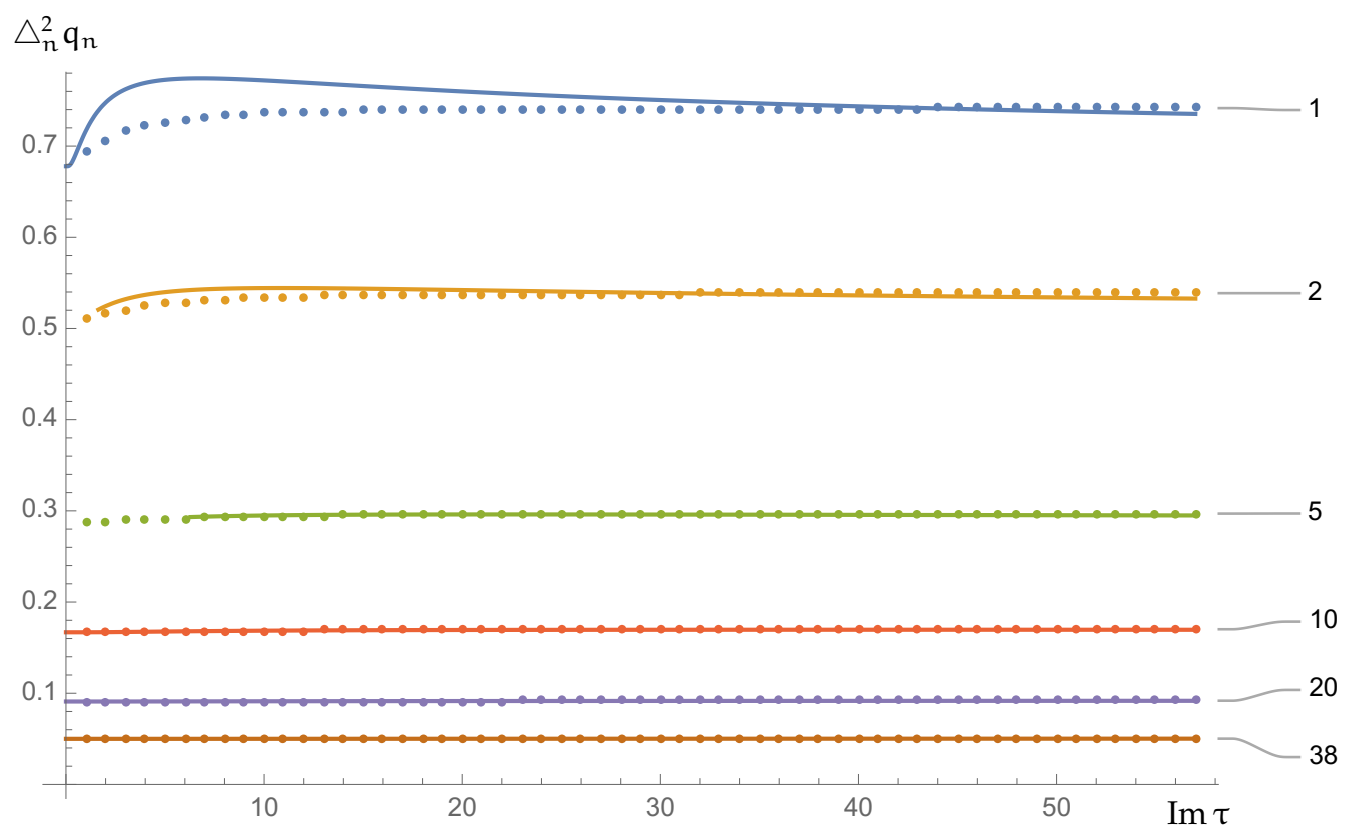

Figure 3. Second difference in $n$ for $\triangle_{n}^{2} q_{n}^{(\mathrm{loc})}$ (dots) and for $\triangle_{n}^{2}\left(q_{n}^{\mathrm{EFT}}+A_{1} e^{-A_{2} \sqrt{n / \operatorname{Im} \tau}}\right)$ (continuous lines) as function of $\operatorname{Im} \tau$ at fixed values of $n$. The exponential term seems to account for most of the discrepancy at small values of $n$ (compare with figure 1 ).

$n$-dependent linear differential equation,

$$
\frac{d^{2}}{d n^{2}} \delta_{n}=\circ \circ \circ
$$

This equation is the well-known ( $\circ \circ)$ equation, whose solution is a linear combination of the two solutions:

$$
\mathcal{S}_{A}(n) \equiv \circ \circ \circ
$$

and

$$
\mathcal{S}_{B}(n) \equiv \circ \circ \circ
$$

Large $\mathcal{J}$ to larger $\mathcal{J}$. Now we can try to understand how small perturbations of initial data, propagate in the asymptotic large- $\mathcal{J}$ regime from large $\mathcal{J}$ to larger $\mathcal{J}$. With $n_{i} \lesssim 1$, we can match the perturbation of the initial conditions precisely with the solutions (5.4), (5.5) to the well-known ( $\circ \circ \circ$ ) equation (5.3).

We have

$$
\delta_{n}=\mathbf{C}_{A} \mathcal{S}_{A}(n)+\mathbf{C}_{B} \mathcal{S}_{B}(n) .
$$

Imposition of $\boldsymbol{S}$-duality symmetry. Let us now consider the case where the theory possesses and $S$-duality symmetry.

First, we note that this is equivalent to the statement that $\exp \left\{q_{n}\right\}$ is a (nonholomorphic) modular form of degree $(\circ \circ \circ) \Delta_{\mathcal{O}}+(\circ \circ \circ) \alpha$ as a function of $\tau, \bar{\tau}$. 


\section{Discussion}

In this article, we have studied $\mathcal{N}=2$ superconformal field theories with a one-complexdimensional Coulomb branch in a sector of fixed and large $R$-charge $\mathcal{J}$. Making use of the resulting effective field theory on the Coulomb branch at large $\mathcal{J}$, we have improved the results of [1], giving the $1 / \mathcal{J}$ expansion of the two-point functions $\mathcal{Y}_{n}=$ $|x-y|^{2 n \Delta_{\mathcal{O}}}\left\langle(\mathcal{O}(x))^{n}(\overline{\mathcal{O}}(y))^{n}\right\rangle$ to all orders. The absence of higher-order $F$-terms in our EFT on the Coulomb branch plays a critical role: it implies that the effective action on the Coulomb branch is given by the tree-level effective kinetic term, the supersymmetrized wz term for the spontaneously broken Weyl invariance, and unknown D-terms which do not affect correlation functions of chiral primaries.

The first term in the large- $\mathcal{J}$ expansion is computed explicitly from the EFT in terms of Feynman diagrams. Then, we observe that for theories with a marginal coupling, we can compute all higher terms using recursion relations, arguing from the EFT that the higher terms do not depend on the marginal coupling.

Even though the recursion relations we solve apply only to SCFTs with a marginal coupling, we find effective recursion relations in the EFT for any theory with a one-dimensional Coulomb branch, whether it has a marginal coupling constant or not. Based on this we argue that the correlators for any four-dimensional $\mathcal{N}=2$ SCFT with a one-dimensional Coulomb branch have a universal large- $\mathcal{J}$ behavior given by

$$
q_{n} \simeq \mathcal{J} \mathbf{A}+\mathbf{B}+\log (\Gamma(\mathcal{J}+\alpha+1)),
$$

where $\mathbf{A}$ and $\mathbf{B}$ are theory-dependent constants and the $\simeq$ indicates the presence of nonuniversal corrections that are exponentially small in $\mathcal{J}$.

We discuss a number of concrete examples, such as $s u(2) \mathcal{N}=2$ SQCD in four dimensions with four flavors, which allows us a direct cross-check against numerical localization computations, verifying our results at high accuracy and allowing us even to see the leading exponential corrections to the asymptotic large-charge expansion.

The results of this article highlight once more the universal applicability of the largequantum number expansion. The constraints imposed on the models by supersymmetry and working at fixed large charge conspire and allow us to obtain analytic results of an unprecedented precision.

Also the importance of the underlying vacuum structure for the large-quantum number expansion is becoming increasingly obvious. The EFTs at fixed charge display a universal behavior depending on the nature of the ground state manifold, as evidenced by the classes of models studied so far with either a unique ground state, such as the $O(n)$ vector models and the $W=\Phi^{3}$ SCFT $[3,4]$, and the $\mathcal{N}=2$ SCFTs with a one-dimensional Coulomb branch discussed in [1] and this article.

There are three obvious directions in which the present work can and should be extended.

- First, it would be very valuable to generalize our results to correlation functions of operators in chiral rings of higher-dimensional Coulomb branches. These correlation 
functions likely lack the degree of universality seen in the present work, because the Coulomb branch EFTs in rank greater than one, contain non-Goldstone excitations. Nonetheless, one would expect holomorphy and symmetries to sharply constrain the possible higher-derivative F-terms, and thus lead to many relations among correlators at large J.

- Second, correlation functions at large SU(2) R-charge could be computed using EFT methods and related to computations by exact methods such as the ones used in [37].

- Third, the leading exponentially small correction to the power-law correlators in the $1 / J$ expansion is quite interesting. Numerical analysis and comparison with the universal power-law terms suggest strongly that the contribution has an interpretation in terms of propagation of a massive particle over macroscopic distance on the infrared scale. It would be illuminating to identify the particle and its semiclassical trajectory, with a goal of matching the exponent and prefactor calculated numerically in the case of $\mathcal{N}=2 \mathrm{sQCD}$ and generalizing the form of the exponentially small correction to the case of non-Lagrangian theories.

Another important future direction is to gain a more intrinsic understanding of the universality of the $1 / J^{m}$ power-law corrections. In the present paper, we have proven our universal formula directly for superconformal gauge theories with marginal coupling, and we have used an indirect argument to show the formula must hold for all theories of rank one, including non-Lagrangian SCFTs. We have also independently computed an infinite series of coefficients directly in the Coulomb-branch EFT, supporting the prediction of the universal formula.

We note that the recursion relations for Coulomb branch correlators in $\mathcal{N}=2$ gauge theories are a particular kind of consistency condition among OPE coefficients, conceptually similar to the conformal bootstrap equations. The simplification of the recursion relations for power-law corrections from differential equations to algebraic equations, is intriguing and suggests the possibility of a simpler derivation within the Coulomb-branch EFT itself, avoiding the need to consider UV-completions of the EFT with marginal coupling. A "algebraic version" of the recursion relations in EFT, not depending on the existence of a marginal coupling, would have a closer formal similarity to the bootstrap equations, with only a finite number of operators exchanged in either channel.

So far, the abstract "bootstrap" approach to CFT has been unable to prove the largecharge behavior of CFT data, that is straightforwardly visible in the EFT picture. ${ }^{3}$ It would be draw a more precise connection between the "EFT version" of the recursion relations, and the conformal bootstrap equations, to derive some aspects large-charge CFT data for higher-rank $\mathcal{N}=2$ theories, $\mathcal{N}=1$ theories, and for theories without vacuum manifolds, such as the $O(2)$ model.

\section{Acknowledgments}

The authors would like to thank Ben Heidenreich for discussions and Zohar Komargodski, Gabriele Tartaglino-Mazzucchelli and Antoine Van Proeyen for correspondence. DO and

\footnotetext{
${ }^{3}$ Though see [38] for progress in this direction.
} 
SR would like to thank the Kavli IPMU for hospitality during part of this work. The work of SH is supported by the World Premier International Research Center Initiative (WPI Initiative), MEXT, Japan; by the JSPS Program for Advancing Strategic International Networks to Accelerate the Circulation of Talented Researchers; and also supported in part by JSPS KAKENHI Grant Numbers JP22740153, JP26400242. The authors particularly thank the WPI Initiative and Kavli IPMU for early funds supporting the meetings that generated this work. SH thanks the University of Bern, the University of Torino, the Burke Institute at Caltech and the Galileo Galilei Institute for hospitality while this research was in progress. SM and MW acknowledge the support by JSPS Research Fellowships for Young Scientists. DO acknowledges partial support by the NCCR 51NF40-141869 "The Mathematics of Physics" (SwISSMAP). The work of SR is supported by the Swiss National Science Foundation (SNF) under grant number PP00P2_157571/1.

\section{A Solving the recurrence equation}

In $[15-18,29]$ it was observed that the correlations functions that we are interested in obey the Toda lattice equation

$$
\partial \bar{\partial} q_{n}(\tau, \bar{\tau})=\exp \left[q_{n+1}(\tau, \bar{\tau})-q_{n}(\tau, \bar{\tau})\right]-\exp \left[q_{n}(\tau, \bar{\tau})-q_{n-1}(\tau, \bar{\tau})\right] .
$$

In this appendix we want to show how to solve this equation using the extra information coming from the EFT about the $\tau$ dependence of the asymptotic expansion of $q_{n}$ for large $n$.

First, it is convenient to rewrite the second-order equation as a system of two first-order equations [39]:

$$
\left\{\begin{array}{l}
\partial P_{n}(\tau, \bar{\tau})=P_{n}(\tau, \bar{\tau})\left(Q_{n}(\tau, \bar{\tau})-Q_{n-1}(\tau, \bar{\tau})\right) \\
\bar{\partial} Q_{n}(\tau, \bar{\tau})=P_{n+1}(\tau, \bar{\tau})-P_{n}(\tau, \bar{\tau})
\end{array}\right.
$$

where

$$
Q_{n}(\tau, \bar{\tau})=\partial q_{n}(\tau, \bar{\tau}), \quad P_{n}(\tau, \bar{\tau})=\exp \left[q_{n}(\tau, \bar{\tau})-q_{n-1}(\tau, \bar{\tau})\right]
$$

In section 2 we have seen that the dependence of $q_{n}(\tau, \tau)$ on $\tau$ is at most affine (i.e. only the constant and linear in $n$ terms depend on $\tau$ ). We can separate this by writing

$$
q_{n}(\tau, \bar{\tau})=n f(\tau, \bar{\tau})+k_{0}(\tau, \bar{\tau})+M_{n} .
$$

The variables $Q_{n}$ and $P_{n}$ then read

$$
\begin{aligned}
Q_{n}(\tau, \bar{\tau}) & =n \partial f(\tau, \bar{\tau})+\partial k_{0}(\tau, \bar{\tau}), \\
P_{n}(\tau, \bar{\tau}) & =e^{f(\tau, \bar{\tau})} \exp \left[M_{n}-M_{n-1}\right]=e^{f(\tau, \bar{\tau})} \Lambda_{n} .
\end{aligned}
$$

With this ansatz the first equation in eq. (A.2) is identically satisfied and we only need to solve

$$
n \partial \bar{\partial} f(\tau, \bar{\tau})+\partial \bar{\partial} k_{0}(\tau, \bar{\tau})=e^{f(\tau, \bar{\tau})}\left(\Lambda_{n+1}-\Lambda_{n}\right) .
$$


If we isolate the terms that do not depend on $\tau, \bar{\tau}$ we can rewrite the equation as the system

$$
\begin{aligned}
\partial \bar{\partial} f(\tau, \bar{\tau}) & =2 A e^{f(\tau, \bar{\tau})}, \\
\partial \bar{\partial} k_{0}(\tau, \bar{\tau}) & =B e^{f(\tau, \bar{\tau})}, \\
\Lambda_{n+1}-\Lambda_{n} & =2 A n+B,
\end{aligned}
$$

where $A$ and $B$ are constants. We see that $f(\tau, \bar{\tau})$ obeys the Liouville equation (A.8) on a hyperbolic plane of Gaussian curvature $-4 A$, and it sources the Poisson equation (A.9) satisfied by $k_{0}(\tau, \bar{\tau})$.

The equation for $\Lambda_{n}$ is easily solved and gives

$$
\Lambda_{n}=A n(n-1)+B n+C^{\prime}=A\left(n-n_{+}\right)\left(n-n_{-}\right),
$$

where $C^{\prime}$ is an integration constant and $n_{ \pm}$are two numbers that satisfy

$$
n_{+}+n_{-}=1-\frac{B}{A}, \quad n_{+} n_{-}=\frac{C^{\prime}}{A} .
$$

Using this expression we can solve for $M_{n}$ :

$$
e^{M_{n}-M_{n-1}}=\Lambda_{n}
$$

and find

$$
M(n)=D+n \log A+\log \left[\Gamma\left(n-n_{-}+1\right) \Gamma\left(n-n_{+}+1\right)\right]
$$

where $D$ is an integration constant.

Let us now consider the $\tau$-dependent equations. The Liouville equation (A.8) for $f(\tau, \bar{\tau})$ admits the general solution

$$
e^{f(\tau, \bar{\tau})}=\frac{1}{A} \frac{|\partial \phi(\tau)|^{2}}{\left(1-|\phi(\tau)|^{2}\right)^{2}},
$$

where $\phi(\tau)$ is a meromorphic function. Now that we have solved for $f(\tau, \bar{\tau})$, we can recast the equation for $k_{0}(\tau, \bar{\tau})$ as a Laplace equation:

$$
\partial \bar{\partial}\left(k_{0}(\tau, \bar{\tau})+\frac{B}{2 A} f(\tau, \bar{\tau})\right)=0
$$

so that $k_{0}(\tau, \bar{\tau})$ is given by

$$
k_{0}(\tau, \bar{\tau})=-\frac{B}{2 A} f(\tau, \bar{\tau})+\psi(\tau)+\bar{\psi}(\bar{\tau})
$$

We can now collect our results and write the final expression for $q_{n}(\tau, \bar{\tau})$ :

$$
\begin{aligned}
q_{n}(\tau, \bar{\tau})= & n f(\tau, \bar{\tau})+k_{0}(\tau, \bar{\tau})+M_{n} \\
= & n(f(\tau, \bar{\tau})+\log A)+k_{0}(\tau, \bar{\tau})+D \\
& +\log \left[\Gamma\left(n-n_{+}+1\right) \Gamma\left(n-n_{-}+1\right)\right] .
\end{aligned}
$$


Our solution depends on the constants, $n_{+}, n_{-}$. They can be fixed in terms of the anomaly coefficient $\alpha$ by comparing the large- $n$ expansion of $q_{n}(\tau, \bar{\tau})$ with the results of the EFT. Expanding the gamma function in the expression in eq. (A.18):

$$
\begin{array}{r}
q_{n}(\tau, \bar{\tau})=n(f(\tau, \bar{\tau})+\log A-2)+k_{0}(\tau, \bar{\tau})+D+\log (2 \pi)-\left(n_{+}+n_{-}-1\right) \log n+ \\
+\frac{1+3 n_{+}\left(n_{+}-1\right)+3 n_{-}\left(n_{-}-1\right)}{6 n}+\ldots
\end{array}
$$

In [1] it was shown that the coefficient of the term $\log (n)$ is $-(\alpha+1 / 2)$, moreover in eq. (2.37) we have found the general form of the $\mathcal{O}(1 / n)$ term as function of $\alpha$. We can use these two conditions to eliminate the constants $n_{ \pm}$:

$$
\left\{\begin{array}{l}
-\left(n_{+}+n_{-}-1\right)=\alpha+\frac{1}{2} \\
\frac{1}{6}\left(1+3 n_{+}\left(n_{+}-1\right)+3 n_{-}\left(n_{-}-1\right)\right)=\frac{1}{4}\left(\alpha^{2}+\frac{\alpha}{2}+\frac{1}{6}\right),
\end{array}\right.
$$

which gives us $n_{ \pm}$as functions of $\alpha$ :

$$
n_{-}=-\frac{\alpha}{2}, \quad n_{+}=\frac{1}{2}-\frac{\alpha}{2} .
$$

This allows us to use the duplication formula for the gamma function and to rewrite $q_{n}(\tau, \bar{\tau})$ in terms of the super-WZ coefficient $\alpha$ :

$$
q_{n}(\tau, \bar{\tau})=2 n \mathbf{A}(\tau, \bar{\tau})+\mathbf{B}(\tau, \bar{\tau})+\log (\Gamma(2 n+\alpha+1)),
$$

where we have collected all the $\tau$ dependence and constants in the two functions $\mathbf{A}$ and $\mathbf{B}$. From this expression we can compute explicitly the large- $\mathcal{J}$ expansion of $q_{n}$ for example in the case of $\mathcal{N}=2 \mathrm{SQCD}$ where $\mathcal{J}=2 n$. We see that the coefficient of $\mathcal{J}^{-m}$ is proportional to the Bernoulli polynomial $B_{m+1}(\alpha+1)$ [40]:

$$
\begin{aligned}
q_{n}(\tau, \bar{\tau})=\mathcal{J} \log (\mathcal{J})+\left(\alpha+\frac{1}{2}\right) \log (\mathcal{J}) & +(\mathbf{A}-1) \mathcal{J}+\mathbf{B}+\log \sqrt{2 \pi} \\
& +\sum_{m=1}^{N} \frac{(-)^{m+1} B_{m+1}(\alpha+1)}{m(m+1) \mathcal{J}^{m}}+\mathcal{O}\left(\frac{1}{\mathcal{J}^{N+1}}\right) .
\end{aligned}
$$

As is well known, the expansion in the last equation is asymptotic and there are corrections of order $\exp [-2 \pi \mathcal{J}]$ that are subdominant with respect to the correction that we have discussed in section 2.5 .

\section{B $\mathcal{N}=2$ supersymmetrization of the Weyl anomaly action}

The WZ term for the Weyl anomaly is given in [22]. An $\mathcal{N}=1$ supersymmetrization of this term was given in [24]. This term it not the unique supersymmetrization preserving $\mathcal{N}=1$ superconformal symmetry: alternate supersymmetrizations of the term can be obtained by adding $\mathcal{N}=1$ superconformally-invariant terms to the action, for instance involving a $\mathcal{N}=1$ superconformal action for the gauge fields. 
Using the extended $\mathcal{N}=2$ superconformal invariance as an input simplifies the matter: as pointed out in [41] there is a unique effective term in the Coulomb-branch dynamics of $\mathcal{N}=2$ superconformal theories in four dimensions; since the super-wz term contains the ordinary wz term in eq. (2.9) for Weyl invariance, this fixes the coefficient unambiguously as well.

In $\mathcal{N}=2$ superspace, the term can be written formally as a full-superspace integral

$$
\mathcal{L}_{\mathcal{N}=2 \text { super-WZ }}=(\text { constant }) \times \int \mathrm{d}^{4} \theta \mathrm{d}^{4} \bar{\theta} \log (\Phi / \mu) \log \left(\Phi^{\dagger} / \mu\right) .
$$

We wish to write this in components, particularly the terms involving the scalar $\phi, \bar{\phi}$ and its derivatives.

The full form of the $\mathcal{N}=2$ super-WZ term is easiest to write in terms of $\mathcal{N}=1$ superfields, as expressed in [42,43]. The $\mathcal{N}=2$ vector multiplet $\Phi$ decomposes into an $\mathcal{N}=1$ superfield $\Phi_{[\mathcal{N}=1]}$ and an $\mathcal{N}=1$ vector multiplet $V$ whose gauge-invariant super-field strength is $\mathcal{W}_{\alpha}$. In $\mathcal{N}=1$ superspace, the form of the term is

$$
\mathcal{L}_{\mathcal{N}=2 \text { super-WZ }}=\int\left(\mathrm{d}^{4} \theta\right)_{\mathcal{N}=1}\left[C_{1} \mathcal{I}_{1}^{[\mathcal{N}=1]}+C_{2} \mathcal{I}_{2}^{[\mathcal{N}=1]}\right]+\left(\text { terms involving } W_{\alpha}\right),
$$

where $C_{1}$ and $C_{2}$ are constants and

$$
\begin{aligned}
& \mathcal{I}_{1} \equiv \frac{1}{\phi_{\mathcal{N}=1} \bar{\phi}_{\mathcal{N}=1}}\left(\partial_{\mu} \phi_{\mathcal{N}=1}\right)\left(\partial^{\mu} \bar{\phi}_{\mathcal{N}=1}\right) \\
& \mathcal{I}_{2} \equiv \frac{1}{\phi_{\mathcal{N}=1} \bar{\phi}_{\mathcal{N}=1}}\left(\epsilon^{\alpha \beta} D_{\alpha} D_{\beta} \phi_{\mathcal{N}=1}\right)\left(\epsilon^{\dot{\alpha} \dot{\beta}} \bar{D}_{\dot{\alpha}} \bar{D}_{\dot{\beta}} \bar{\phi}_{\mathcal{N}=1}\right) .
\end{aligned}
$$

The $D_{\alpha}$ and $\bar{D}_{\dot{\alpha}}$ are the spinorial superspace covariant derivatives.

In addition to the lowest component $\phi$, the $\mathcal{N}=1$ superfield contains fermions and a complex auxiliary field $F$, which is the lowest component of $D^{2} \Phi$. In a generic $\mathcal{N}=1$ action, we would have to keep track of terms coupling $F$ to $\phi$ and $\bar{\phi}$ : after eliminating the auxiliary fields, these would become terms of order $\alpha^{2}$ which could contribute to the classical action. However such terms cannot appear in a superconformal effective action for a vector multiplet alone: the real and imaginary parts of $F$ transform together with the real auxiliary field $D$ of the $\mathcal{N}=1$ vector multiplet, as a triplet under the $\mathrm{SU}(2) R$ symmetry in the superconformal algebra, and any term coupling linearly to $F$ or $D$, would have to be a triplet as well. Since $\phi$ and $A_{\mu}$ are invariant under the SU(2) R-symmetry, the only way to build a scalar term coupling linearly in the auxiliary fields, would be to include at least two fermions. Therefore the values of the auxiliary fields after eliminating them by their EOM, can have no component involving only the scalars and photon. We are interested in this section only in the classical action, and the fermions only contribute quantum mechanically. So we can treat the auxiliary field $F$ as zero for purposes of writing down the action for the scalars alone.

For the same reason, it will be unnecessary to keep track of any component terms involving the $\mathcal{N}=1$ vector superfield: in the classical solutions relevant to the two-point function of $\phi$, the gauge field strength vanishes, and so all terms involving the $\mathcal{N}=1$ vector superfield vanish classically and contribute only through their quantum effects. 
So we need only consider the superspace integrals of the two terms $\mathcal{I}_{1,2}$, and in particular only the component terms containing no fermions or auxiliary fields.

With attention restricted to such component terms, the superspace integral of $\mathcal{I}_{2}$ is easiest to compute. In order to obtain a term involving only scalars, we must take the $\bar{\theta} \bar{\theta}$ component of $D^{2} \Phi_{\mathcal{N}=1}$ and the $\theta \theta$ component of $\bar{D}^{2} \Phi_{\mathcal{N}=1}^{\dagger}$, which are proportional to $\partial^{2} \phi$ and $\partial^{2} \bar{\phi}$, respectively. So we have

$$
\int \mathrm{d}^{4} \theta_{\mathcal{N}=1} \mathcal{I}_{2} \simeq\left(\text { constant }^{\prime}\right)_{2}|\phi|^{-2}\left(\partial^{2} \phi\right)\left(\partial^{2} \bar{\phi}\right),
$$

where the $\simeq$ denotes the omission of terms involving fermions and auxiliary fields.

The superspace integral of $\mathcal{I}_{1}$ can be evaluated easily using a trick: treat $\partial_{\mu} \phi_{\mathcal{N}=1}$ and its conjugate as independent superfields $G_{\mu}, G_{\mu}^{\dagger}$, and write the superspace integrand as a Kahler potential for the five superfields $\chi^{A} \in\left\{\phi_{\mathcal{N}=1}, G_{\mu}\right\}$ and their conjugates. So

$$
\mathcal{I}_{1}=\mathcal{K}\left(\chi, \chi^{\dagger}\right)=\left|\phi_{\mathcal{N}=1}\right|^{-2} \eta^{\mu \nu} G_{\mu} G_{\nu}^{\dagger} .
$$

Then the superspace integral is given by the usual formula written in terms of the Kahler potential,

$$
\begin{aligned}
\int\left(\mathrm{d}^{4} \theta\right)_{\mathcal{N}=1} \mathcal{I}_{1} \simeq & \mathcal{K}_{, \chi^{A} \chi^{B \dagger}} \partial_{\nu} \chi^{A} \partial^{\nu} \chi^{B \dagger} \\
= & \mathcal{K}_{, \phi \bar{\phi}} \partial_{\nu} \phi \partial^{\nu} \bar{\phi}+\mathcal{K}_{, \phi G_{\mu}^{\dagger}} \partial_{\nu} \phi \partial^{\nu} G_{\mu}^{\dagger}+\mathcal{K}_{, G_{\mu} \bar{\phi}} \partial^{\nu} G_{\mu} \partial_{\nu} \bar{\phi} \\
& +\mathcal{K}_{, G_{\mu} G_{\rho}^{\dagger}} \partial^{\nu} G_{\mu} \partial_{\nu} G_{\rho}^{\dagger} \\
= & |\phi|^{-4} G^{\mu} G_{\mu}^{\dagger} \partial_{\nu} \phi \partial^{\nu} \bar{\phi}-\phi^{-2} \bar{\phi}^{-1} G^{\mu} \partial_{\nu} \phi \partial^{\nu} G_{\mu}^{\dagger} \\
& -\bar{\phi}^{-2} \phi^{-1} G_{\mu}^{\dagger} \partial_{\nu} \bar{\phi} \partial^{\nu} G^{\mu}+|\phi|^{-2} \partial^{\mu} G^{\nu} \partial_{\mu} G_{\nu}^{\dagger} \\
= & |\phi|^{-4}(\partial \phi \partial \phi)^{2}-\phi^{-2} \bar{\phi}^{-1}\left(\partial^{\mu} \partial^{\nu} \bar{\phi}\right)\left(\partial_{\mu} \phi\right)\left(\partial_{\nu} \phi\right) \\
& -\bar{\phi}^{-2} \phi^{-1}\left(\partial^{\mu} \partial^{\nu} \phi\right)\left(\partial_{\mu} \bar{\phi}\right)\left(\partial_{\nu} \bar{\phi}\right)+|\phi|^{-2}\left(\partial^{\mu} \partial^{\nu} \phi\right)\left(\partial_{\mu} \partial_{\nu} \bar{\phi}\right) .
\end{aligned}
$$

Rewriting the two terms with the substitution

$$
\phi=\mu \exp \{-\tau-i \beta\}, \quad \bar{\phi}=\mu \exp \{-\tau+i \beta\},
$$

we get

$$
\int \mathrm{d}^{4} \theta_{\mathcal{N}=1} \mathcal{I}_{1}=(\text { constant }) \times\left(\partial^{\mu} \partial^{\nu} \tau\right)\left(\partial_{\mu} \partial_{\nu} \tau\right)+(\text { fermions and auxiliary }),
$$

and

$$
\begin{array}{r}
\int\left(\mathrm{d}^{4} \theta\right)_{\mathcal{N}=1} \mathcal{I}_{2}=(\text { constant }) \times\left[\left(\partial^{\mu} \partial^{\nu} \tau\right)\left(\partial_{\mu} \partial_{\nu} \tau\right)-2\left(\partial^{\mu} \partial^{\nu} \tau\right)\left(\partial_{\mu} \tau\right)\left(\partial_{\nu} \tau\right)+(\partial \tau)^{4}\right] \\
+(\text { fermions and auxiliary })
\end{array}
$$

Modulo total derivatives, and dropping the terms involving fermions and auxiliary fields, this is

$$
\int\left(\mathrm{d}^{4} \theta\right)_{\mathcal{N}=1} \mathcal{I}_{1}=(\text { constant }) \times\left(\partial^{2} \tau\right)^{2}+(\text { fermions and auxiliary }),
$$


and

$$
\begin{aligned}
\int\left(\mathrm{d}^{4} \theta\right)_{\mathcal{N}=1} \mathcal{I}_{2}=(\text { constant }) \times\left[\left(\partial^{2} \tau\right)^{2}-2(\partial \tau)^{2}\left(\partial^{2} \tau\right)\right. & \left.+(\partial \tau)^{4}\right] \\
& +(\text { fermions and auxiliary }) .
\end{aligned}
$$

The coefficients are given in $[42,43]$, but it is simple to see what they must be. The relative coefficient between the two terms must be -1 : when $\beta$ is set to a constant, we must recover the usual WZ action for the Weyl symmetry given in [22], which contains no term proportional to $\left(\partial^{2} \tau\right)^{2}$. The absolute coefficient is also given by matching with [22], so that the purely dilaton-dependent part of the super-WZ term is equal to the KS dilaton action:

$$
\text { const. } \times \int\left(\mathrm{d}^{4} \theta\right)_{\mathcal{N}=1}\left[\mathcal{I}_{1}-\mathcal{I}_{2}\right]=\text { const. } \times\left[(\partial \tau)^{4}-2(\partial \tau)^{2}\left(\partial^{2} \tau\right)\right]
$$

where the constant is fixed by the anomaly. This fixes the two coefficients; so we conclude the dilaton and axion part of the super-wz term, and we get

$$
\begin{array}{r}
\mathcal{L}_{\text {super-WZ }}^{\text {(Euclidean })}=+2(\Delta a)^{[\mathrm{KS}]}\left[(\partial \tau)^{4}-2\left(\partial^{2} \tau\right)(\partial \tau)^{2}+2\left(\partial^{2} \tau\right)(\partial \beta)^{2}-4(\partial \tau \cdot \partial \beta)\left(\partial^{2} \beta\right)\right. \\
\left.-2(\partial \tau)^{2}(\partial \beta)^{2}+4(\partial \tau \cdot \partial \beta)^{2}+(\partial \beta)^{4}\right],
\end{array}
$$

where we have evaluated the term in flat space, and dropped terms involving the gauge field, fermions, and auxiliary fields. Note that this agrees with the flat space expression given in [24].

We would like to write this action in other conformal frames, such as the sphere $S^{4}$ or the cylinder $S^{3} \times \mathbb{R}$. In order to do this, we need to include the appropriate curvature couplings that give the full action an appropriate transformation law. The transformation law for the curved-space super-WZ action must obey is itself nontrivial, because the $\mathcal{N}=2$ super-WZ action should not be conformally invariant: indeed, it must have a nonvanishing additive transformation under a Weyl transformation, in order to reproduce the anomalous quantum transformation of the logs of the determinants for the massive fields which have been integrated out. However it can be decomposed into the KS action (2.9) itself, plus a remainder term. The former has the anomalous transformation law dictated by the WZ consistency condition, and so the remainder must be invariant under Weyl transformations.

On flat space, the action breaks up as:

$$
S[\tau, \beta, g]=S_{[\mathrm{KS}]}[\tau, g]+S_{[\text {remainder }]}[\tau, g],
$$

where

$$
\begin{aligned}
S_{[\mathrm{KS}]}[\tau, g] \equiv & +2(\Delta a)^{[\mathrm{KS}]}\left[(\partial \tau)^{4}-2\left(\partial^{2} \tau\right)(\partial \tau)^{2}\right] \\
S_{[\text {remainder }]}[\tau, g] \equiv & +2(\Delta a)^{[\mathrm{KS}]}\left[+2\left(\partial^{2} \tau\right)(\partial \beta)^{2}-4(\partial \tau \cdot \partial \beta)\left(\partial^{2} \beta\right)\right. \\
& \left.-2(\partial \tau)^{2}(\partial \beta)^{2}+4(\partial \tau \cdot \partial \beta)^{2}+(\partial \beta)^{4}\right]
\end{aligned}
$$


The remainder term is covariant. For any conformally flat space, the covariantization is unique, and given by turning the flat metric into the dressed hatted metric:

$$
\hat{g}_{\mu \nu} \equiv \exp [-2 \tau] g_{\mu \nu}
$$

On flat space, we have already worked out the remainder, which fixes its covariantization. The covariant action for the axiodilaton alone in a general conformally flat metric, has been given in [24]. Therefore the axiodilaton part of the remainder term is given by the difference between this action and the KS dilation action. In the $\mathcal{N}=2$ theory, there are also terms involving gauge field strengths and fermions, which we omit, because we will not need them: the gauge fields and fermions make no contribution up to and including order $1 / \mathcal{J}$, and we have derived the higher power-law corrections on general grounds without the need to use the other terms in the action explicitly.

The covariantization of the remainder term is given in [24] as

$$
\mathcal{L}_{\substack{\text { Remainder } \\ \text { BEO }}}^{(\text {Lorentzian })} \equiv-4\left(\Delta a^{[\mathrm{KS}]}\right) \frac{\sqrt{-\hat{g}}}{\sqrt{-g}}\left[\left(\hat{R}^{\mu \nu}-\frac{1}{6} \hat{R} \hat{g}^{\mu \nu}\right) \nabla_{\mu} \beta \nabla_{\nu} \beta+\frac{1}{2}\left(\hat{g}^{\mu \nu} \nabla_{\mu} \beta \nabla_{\nu} \beta\right)^{2}\right] .
$$

In four dimensions the Riemann curvature is given by a sum of the Ricci tensor and Weyl tensor, and so any term vanishing in conformally flat space, must be proportional to at least one power of the Weyl tensor and its derivatives. Since we are only ever considering conformally flat geometries in this paper, we will henceforth drop all terms involving the Weyl tensor. Therefore on a general space we have

$$
\mathcal{L}_{\text {Remainder }}^{(\text {Lorentzian })}=\mathcal{L}_{\substack{\text { Remainder } \\ \text { BEO }}}^{(\text {Lorentian }}+(\text { covariant terms involving the Weyl tensor }) .
$$

and of course the pure Weyl-anomaly term is given by the KS-action given in eq. (2.9).

So, all in all we have

$$
\begin{aligned}
S_{\text {super-WZ, curved }}^{(\text {lorentzian })} & -\Delta a^{[\mathrm{KS}]} \int \mathrm{d}^{4} x \sqrt{-g}\left[\tau E_{4}^{[\mathrm{KS}]}+\left(4\left(R^{\mu \nu}-\frac{1}{2} R g^{\mu \nu}\right) \nabla_{\mu} \tau \nabla_{\nu} \tau\right.\right. \\
& \left.\left.-2(\nabla \tau)^{2}\left(2 \square \tau-(\nabla \tau)^{2}\right)\right)\right] \\
+ & 4 \sqrt{-\hat{g}}\left[\left(\hat{R}^{\mu \nu}-\frac{1}{6} \hat{R} \hat{g}^{\mu \nu}\right) \nabla_{\mu} \beta \nabla_{\nu} \beta+\frac{1}{2}\left(\hat{g}^{\mu \nu} \nabla_{\mu} \beta \nabla_{\nu} \beta\right)^{2}\right] \\
+ & (\text { covariant terms invoving the Weyl tensor }) .
\end{aligned}
$$

It is convenient to separate the dependence on the powers of dilaton and axion:

$$
\mathcal{L}_{\text {super-WZ }}^{\text {(Lorentzian) }}=\mathcal{L}_{\tau^{1}}+\mathcal{L}_{\tau^{2}}+\mathcal{L}_{\tau^{3}}+\mathcal{L}_{\tau^{4}}+\mathcal{L}_{\beta^{2}}+\mathcal{L}_{\tau^{1} \beta^{2}}+\mathcal{L}_{\tau^{2} \beta^{2}}+\mathcal{L}_{\beta^{4}}
$$


where

$$
\begin{aligned}
\mathcal{L}_{\tau^{1}} & =-(\Delta a) \tau E_{4}, \\
\mathcal{L}_{\tau^{2}} & =-4(\Delta a)\left[R^{\mu \nu}-\frac{1}{2} \mathrm{Ric}_{4} g^{\mu \nu}\right] \nabla_{\mu} \tau \nabla_{\nu} \tau, \\
\mathcal{L}_{\tau^{3}} & =+4(\Delta a)(\nabla \tau)^{2}\left(\nabla^{2} \tau\right) \\
\mathcal{L}_{\tau^{4}} & =-2(\Delta a)(\nabla \tau)^{4} \\
\mathcal{L}_{\beta^{2}} & =-4(\Delta a)\left[R^{\mu \nu}-\frac{1}{6} \operatorname{Ric}_{4} g^{\mu \nu}\right]\left(\nabla_{\mu} \beta\right)\left(\nabla_{\nu} \beta\right) \\
\mathcal{L}_{\tau^{1} \beta^{2}} & =-8(\Delta a)\left(\nabla^{\mu} \nabla^{\nu} \tau\right) \nabla_{\mu} \beta \nabla_{\nu} \beta, \\
\mathcal{L}_{\tau^{2} \beta^{2}} & =-4(\Delta a)\left[2(\nabla \tau \cdot \nabla \beta)^{2}-(\nabla \tau)^{2}(\nabla \beta)^{2}\right], \\
\mathcal{L}_{\beta^{4}} & =-2(\Delta a)(\nabla \beta)^{4} .
\end{aligned}
$$

\section{Nonexistence of higher-derivative $F$-terms on conformally flat space}

In general $\mathcal{N}=2$ supersymmetric gauge theories, the effective action on the Coulomb branch has higher derivative $F$-terms, of which those with few derivatives have been partially classified by [25-28]. In the case of superconformal gauge theories with rank one, the remarkable simplifications of the dynamics of the Coulomb branch have to do with the absence of such terms.

More precisely, the only half-superspace integrands consistent with superconformal symmetry on a general curved background, are the tree-level kinetic term proportional to $\Phi^{2}$, and terms involving the background Weyl multiplet, which contains the $\mathrm{U}(1)_{\mathrm{R}}$-photon background and the self-dual part of the Weyl tensor.

Vanishing of higher-derivative terms on (superconformally) flat space. Consider the effective action of a single Abelian vector multiplet in a superconformally invariant theory. The symmetries of a $\mathcal{N}=2$ superconformal theory include dilatation invariance and $\mathrm{U}(1) \mathrm{R}$-symmetry, which act on a vector multiplet $\phi$ by rescalings and complex phase rotations respectively, both in the underlying microscopic CFT and in the EFT of the Coulomb branch. The Weyl symmetry acts as

$$
\phi \rightarrow \exp \{\rho\} \phi,
$$

and the $\mathrm{U}(1)_{R}$ acts as

$$
\phi \rightarrow \exp \{i \gamma\} \phi .
$$

One can combine the Weyl and $\mathrm{U}(1)_{R}$ parameters into a single complex parameter $\sigma \equiv$ $-\rho-i \gamma$, which acts as

$$
\phi \rightarrow \exp \{-\sigma\} \phi, \quad \bar{\phi} \rightarrow \exp \{-\bar{\sigma}\} \bar{\phi}
$$


In a superconformal theory it is natural to promote $\sigma$ to a local function of superspace rather than just the $x$ coordinates. In order to preserve the chirality constraint $\bar{Q}_{\dot{\alpha}}^{i} \cdot \phi=0$ we can require $\sigma$ to obey the same chirality constraint $\bar{Q}_{\dot{\alpha}}^{i} \cdot \sigma=0$. Invariance of a superconformal theory under super-Weyl transformations parametrized by a chiral superfield has been studied previously (see [44] and references within for $\mathcal{N}=1$ theories and [45] and references within for $\mathcal{N}=2$ theories).

The chiral superfield Weyl parameter $\sigma$ consists of a complex scalar, fermions, and a vector parameter $\hat{\lambda}_{\mu}$, and other components which act only on the auxiliary fields. The scalar and fermionic members of the parameter superfield implement Weyl, $\mathrm{U}(1)_{R}$, and local supersymmetry transformations, respectively; the $\lambda_{\mu}$ transformations shift the gauge field as

$$
\delta A_{\mu}=\phi \hat{\lambda}_{\mu}
$$

Local transformations are not themselves symmetries of the dynamical fields alone, but can be understood as "spurionic" symmetries, that preserve the action for dynamical variables together with a set of background fields, when the background fields are transformed appropriately. In the case of local dilatation and local $\mathrm{U}(1)_{R}$ transformations, the corresponding background fields are the metric and the $\mathrm{U}(1)_{R}$ gauge field, which transform by Weyl transformations and local $\mathrm{U}(1)_{R}$ gauge transformations, respectively. The $\lambda_{\mu}$-transformations can be thought of as shifting a background antisymmetric tensor field $B_{\mu \nu}$ by a gauge transformation

$$
B_{\mu \nu} \rightarrow(d \hat{\lambda})_{\mu \nu}=\partial_{\mu} \hat{\lambda}_{\nu}-(\mu \leftrightarrow \nu)
$$

There is no unique or canonical formulation of supergravity off-shell, even $\mathcal{N}=1$ SUGRA, and the variety of off-shell formulations of $\mathcal{N}=2$ SUGRA is even larger. The action of minimal super-Weyl invariance on the vector multiplet is necessarily the same in any offshell formalism, since it can be expressed directly in terms of physical currents and their operator products with physical vector multiplet degrees of freedom. So, the transformation of the superfield $\phi$ under a super-Weyl transformation paramerized by the chiral superfield $\sigma$, is independent of the $\mathcal{N}=2$ SUGRA formalism and set of additional compensators and auxiliary fields needed to give a complete off-shell formulation.

Constraints on the EFT from super-Weyl invariance. We observe that the Wilsonian effective action is super-Weyl invariant if the underlying CFT is super-Weyl invariant: the EFT on moduli space inherits this property directly from the CFT. Super-Weyl invariance of the CFT is automatic if the theory is supersymmetric and conformal. Weyl invariance can be seen explicitly at the Lagrangian level for superconformal gauge theories with hypermultiplets and vanishing $\beta$-function; super-Weyl invariance also acts on the vector-multiplet action in a transparent way.

The EFT inherits the super-Weyl invariance of the underlying CFT, so we can now consider what possible terms one might write in a supersymmetric $\mathcal{N}=2$ EFT consistent with super-Weyl invariance.

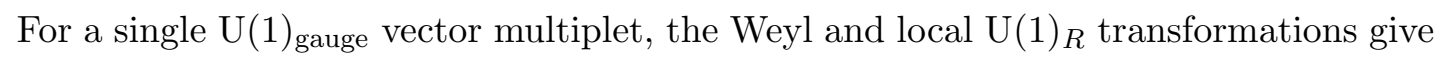
enough freedom to set the complex scalar $\phi$ equal to some fixed nonzero value, say $\mu$, 
everywhere that it is nonvanishing: by choosing $\sigma=+\log (\phi / \mu)$ we can fix the "gauge" $\phi=\mu$.

The fermions $\psi_{\alpha}^{i}$ in the Abelian vector multiplet are superpartners of $\phi$, and supersymmetry implies that if $\phi-\mu$ can be made to vanish with a local transformation, then $\psi_{\alpha}^{i}$ can be made to vanish as well. And, indeed, superconformal transformations can be promoted to local transformations as well: by integrating the supercurrents against general functions $\eta_{\alpha}^{i}(x)$ of space, we have enough freedom to set to zero the fermions $\psi_{\alpha}$ at the cost of turning on a nonzero but flat background for the (spurionic) gravitini.

The freedom to make $\hat{\lambda}_{\mu}$-transformations (C.5) allows us to set the gauge field to zero as well, and so the entire vector multiplet in the EFT can be gauged away. It follows that there can be no super-Weyl-invariant terms containing only the metric and no background curvatures.

Certainly there may be many terms involving background curvatures, but we are considering only the maximally supersymmetric background $\mathbb{R}^{4}$ and backgrounds equivalent to it such as the sphere $S^{4}$ and the cylinder $S^{3} \times \mathbb{R}$. We therefore need only consider couplings involving the Ricci curvature and its derivatives, since the Weyl curvature and R-symmetry gauge flux vanish in the backgrounds we consider.

For D-terms there are many such terms one can construct: the dressed metric $\hat{g}_{\mu \nu} \equiv$ $|\phi|^{2} g_{\mu \nu}$ is Weyl-invariant and its superspace extension is super-Weyl-invariant by construction. So any term constructed from these has Weyl weight zero and is suitable for addition to the action as a $D$-term (i.e., full $\mathcal{N}=2$ superspace $d^{4} \theta d^{4} \bar{\theta}$ integrand) consistent with super-Weyl invariance.

The correlators we consider in the present paper are computable by localization and insensitive to $D$-terms; we need therefore consider only super-Weyl-invariant $F$-term contributions to the effective action. ${ }^{4}$

Such terms must be of the form

$$
\Delta \mathcal{L}=\int d^{4} \theta \phi^{2} \mathcal{I}_{0}
$$

where $\mathcal{I}_{0}$ is a super-Weyl-invariant term that is also a chiral primary field, i.e., annihilated by all the $\bar{D}_{\dot{\alpha}}^{i}$ superderivatives. As we have pointed out above, such terms must be constructed from Ricci curvatures of the hatted metric. However the hatted metric is not a chiral field, nor is the Ricci curvature or any of its derivatives. One can see this easily from its definition: acting with $\bar{D}_{\dot{\alpha}}^{i}$ on $\hat{g}_{\mu \nu}$ gives

$$
\bar{D}_{\dot{\alpha}}^{i}\left(\hat{g}_{\mu \nu}\right)=\omega \hat{g}_{\mu \nu}, \quad \omega \equiv \bar{\psi}_{\dot{\alpha}}^{i} / \bar{\phi}
$$

In other words, even though $\hat{g}_{\mu \nu}$ is Weyl-invariant, acting with the antichiral supersapce derivative on $\hat{g}_{\mu \nu}$ is equivalent to infinitesimally Weyl-transfroming the hatted metric by a

\footnotetext{
${ }^{4}$ In addition to the familiar $D$-terms and $F$-terms, $\mathcal{N}=2$ supersymmetric effective theories with hypermultiplets may have terms that can be represented as $\frac{3}{4}$-superspace integrals but not true $F$-terms. Some such terms have been worked out in [25-28]. However we can restrict our attention to theories with only a pure Coulomb branch and no massless neutral hypers, rather than an enhanced Coulomb branch. For theories with no hypermultiplets we may consider only the usual $F$-terms and $D$-terms.
} 
Weyl parameter proportional to $\bar{\psi}_{\dot{\alpha}}^{i} / \bar{\phi}$, which does not vanish identically, obviously. The only quantities that can be constructed from $\hat{g}_{\mu \nu}$ are exactly the same as the Weyl-invariant quantities that could be constructed from the unhatted background metric $g_{\mu \nu}$, with $g_{\mu \nu}$ replaced by $\hat{g}_{\mu \nu}$. But since these quantities are Weyl-invariant, the replacement has no effect and they are exactly the same as the ones constructed from $g_{\mu \nu}$, that is, the Weyl curvature and various powers of it and its Weyl-covariantized derivatives.

As mentioned earlier, many such terms can be constructed, and would contribute to $F$-terms on a non-conformally-flat background; however for a background with vanishing Weyl curvature, all such terms vanish. We therefore conclude that all higher-derivative $F$-terms vanish identically on a conformally flat background, in the effective theory of a single Abelian vector multiplet. Adding a flat background connection for the $\mathrm{U}(1)_{R^{-}}$ symmetry, allows more terms to be written but does not change the conclusion: there are no superconformally invariant higher-derivative $F$-terms that can be written for a single vector multiplet, even with an R-symmetry connection included, so long as the flux vanishes and the metric is conformally flat.

More comments on the currents. The action of the super-Weyl transformations on the physical fields is generated by currents with protected integer operator dimensions living in a single current multiplet; for the case of dilatations the generating operator is the trace of the stress tensor with dimension 4 and for $\mathrm{U}(1)$ transformations the generating

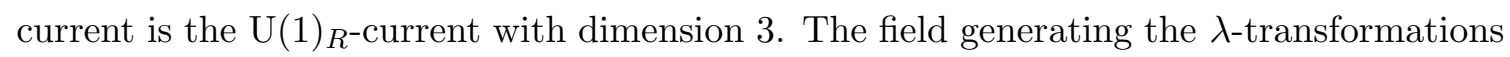
is an antisymmetric tensor of weight 3 (see for instance [46, 47]) which is not a conserved current but whose curl is the weight- 4 topological current that integrates to the central charge $\mathcal{Z}$.

Since these currents are local, they can be integrated against arbitrary functions to generate well-defined local transformations of the fields. This is the physical basis of the super-Weyl transformation: an infinitesimal change of the SUGRA background is equivalent to an infinitesimal transformation of the physical degrees of freedom, which in turn is equivalent to inserting integrated currents into the path integral. For instance an infinitesimal change in the background metric is equivalent to

$$
S \rightarrow S+\int \sqrt{|g|}\left(\delta g_{\mu \nu}\right) T^{\mu \nu}
$$

an infinitesimal change in the R-symmetry gauge connection is equivalent to

$$
S \rightarrow S+\int \sqrt{|g|}\left(\delta \mathcal{A}_{\mu}^{\mathrm{U}(1)_{R}}\right) J_{\mathrm{U}(1)_{R}}^{\mu}
$$

and an infinitesimal change in the antisymmetric tensor background is equivalent to

$$
S \rightarrow S+\int \sqrt{|g|}\left(\delta \mathcal{B}_{\mu \nu}\right) \mathcal{Z}^{\mu \nu}+\text { (c.c.) }
$$

Diffeomorphism and Weyl invariance are equivalent to the statements that $T_{\mu \nu}$ is divergenceless and traceless, respectively; $\mathrm{U}(1)_{\mathrm{R}}$ invariance is equivalent to the statement that $J_{\mathrm{U}(1)_{R}}^{\mu}$ is divergenceless. There is no simple analogous statement about the $\mathcal{Z}$-current, 
which sits in the (short) stress tensor multiplet as an anti-self-dual tensor with conformal dimension 3. At the free-field level, the $\mathcal{Z}$-current is proportional to $\phi F_{\mu \nu}^{(-)}$, where $F^{(-)}$is the anti-self-dual part of the gauge field strength. Its complex conjugate generates $\hat{\lambda}$-transformations on the vector multiplet when integrated against $\hat{\lambda}$.

Unlike the R-current and stress tensor, its divergence does not vanish. Correspondingly, the coupling of $\mathcal{Z}^{\mu \nu}$ to the background $\mathcal{B}_{\mu \nu}$-field is somewhat subtle; the $\hat{\lambda}$ one-form transformations act on other background fields in addition to the $\mathcal{B}_{\mu \nu}$-field. The coupling of the $\mathcal{Z}$-current to the SUGRA background is formalism-dependent, as the $\mathcal{B}$-field is not part of the minimal $\mathcal{N}=2$ SUGRA multiplet and the details have not been worked out in the SUGRA literature. One can infer the physically relevant properties of the coupling by considering the current directly, whose properties are formalism-independent.

The $\mathcal{Z}_{\mu \nu}$ current, which generates the $\lambda_{\mu}$-transformations which shift the gauge field in the vector multiplet, is less well-studied than the other members of its multiplet, the stress tensor and $R$-current. Since the super-Weyl transformation generated in part by $\mathcal{Z}_{\mu \nu}$ plays a role in forbidding higher-derivative $F$-terms for one-dimensional Coulomb-branch EFTs, we comment briefly on properties of this current for the sake of context [46-48].

The $\mathcal{Z}_{\mu \nu}$ current is similar to the line-charge symmetry that shifts the photon in a weakly-coupled Maxwell gauge theory [49, 50], but it is a different sort of current. The linecharge current in four-dimensional Abelian gauge theory has dimension approximately two at weak coupling rather than three, and cannot be exactly conserved unless the dimension is exactly two and Maxwell field is exactly free, in analogy with the parallel Sugawara theorem for spin-one currents in two dimensions [51].

By contrast the $\mathcal{Z}$-current has dimension three and is not divergenceless. Indeed, the divergence of the $\mathcal{Z}$-current contributes to the central charge in the $\mathcal{N}=2$ supersymmetry algebra. That is,

$$
\begin{aligned}
\left\{Q_{\alpha}^{i}, Q_{\beta}^{j}\right\} & =2 \epsilon^{i j} \epsilon_{\alpha \beta} Z, \\
Z & \ni \int d^{3} \mathcal{N}^{\mu} \nabla^{\nu} \mathcal{Z}_{\mu \nu}, \\
\mathcal{Z}_{\mu \nu} & \propto \epsilon_{i j}\left(\left[\sigma_{\mu}, \sigma_{\nu}\right]\right)^{\alpha \beta} \epsilon_{i j} Q_{\alpha}^{i} \cdot Q_{\beta}^{j} \cdot \mathbf{J}_{\text {scalar }},
\end{aligned}
$$

where $\mathbf{J}_{\text {scalar }}$ is the lowest component of the stress tensor multiplet, a scalar primary of dimension $\Delta=2$ transforming trivially under the $R$ symmetry and equal to $\phi \bar{\phi}$ in the Coulomb-branch EFT [46, 47].

In a superconformal $\mathcal{N}=2$ theory without marginal operators, this current is the only contribution to the central charge; there are no other currents of dimension three and the correct quantum numbers to appear in the susy algebra. The normalization of the central charge $Z$ is therefore determined by the three-point function of the current multiplet in such theories, which means its value is fixed entirely by the anomaly coefficients $a$ and $c$. This has interesting implications for the BPS dyon spectrum on the Coulomb branch of non-Lagrangian $\mathcal{N}=2$ SCFT.

In a superconformal $\mathcal{N}=2$ theory with marginal operators, there is a second independent component of the central charge, also a total derivative, of a current which we shall 
call $\mathcal{Y}_{\mu \nu}$ :

$$
\begin{aligned}
Z & \ni \int d^{3} \mathcal{N}^{\mu} \nabla^{\nu} \mathcal{Y}_{\mu \nu}, \\
\mathcal{Y}_{\mu \nu} & =\sum_{A} y_{A} \epsilon_{i j}\left(\left[\sigma_{\mu}, \sigma_{\nu}\right]\right)^{\alpha \beta} \epsilon_{i j} Q_{\alpha}^{i} \cdot Q_{\beta}^{j} \cdot \mathcal{O}^{A},
\end{aligned}
$$

where $A$ runs over all marginal operators $\mathcal{O}^{A}$ and $y_{A}$ can vary over the conformal manifold. All the dependence of the central charge on the marginal directions is through the $\mathcal{Y}$-current contribution.

\section{D $\mathcal{N}=2$ superconformal gauge dynamics with ghost hypermultiplets}

Weyl anomalies and $\beta$-functions for $\mathcal{N}=2$ gauge theory with $G=\mathrm{SU}(2)$. Consider for instance the case of an $\mathcal{N}=2$ gauge theory with $G=\mathrm{SU}(2)$ and ordinary hypermultiplets. A hypermultiplet in a representation $\mathbf{R}$ of $\mathrm{SU}(2)$ contributes to the $\beta$ function as

$$
\beta_{\text {ordinary hypermultiplet in } \mathbf{R}}=+\frac{g_{\mathrm{YM}}^{3}}{16 \pi^{2}} \operatorname{Tr}_{\mathbf{R}}\left(t^{A} t^{A}\right),
$$

where $A=1,2,3$ and the representation matrices $t^{A}$ are taken to be Hermitean and normalized so that the level spacing of $t^{A=3}$ is differences of 1 . So if $\mathbf{R}$ is the $k$-dimensional representation then $t^{3}$ has eigenvalues $\left\{-\frac{k-1}{2},-\frac{k-3}{2}, \cdots,+\frac{k-3}{2},+\frac{k-1}{2}\right\}$, so

$$
\operatorname{Tr}_{\mathbf{R}}\left(t^{A} t^{A}\right)=3 \times \operatorname{tr}_{\mathbf{R}}\left(\left(t^{A=3}\right)^{2}\right)=\frac{k\left(k^{2}-1\right)}{4} .
$$

In terms of the largest eigenvalue $\ell \equiv k-\frac{1}{2}$ of $t^{A=3}$, this is just the dimension $k=2 \ell+1$ of the representation, times the quadratic Casimir $\ell(\ell+1)=\frac{1}{4}\left(k^{2}-1\right)$.

So the $\beta$-function of an ordinary hypermultiplet is

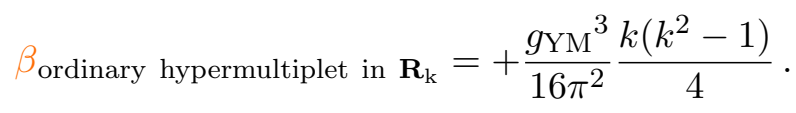

The $\beta$-function in $\mathcal{N}=2$ theories comes entirely from one loop.

Ghost hypermultiplets. If we were to couple hypermultiplets in representation $\mathbf{R}$ with spin-statistics opposite to the usual ones, then the $\beta$ function would be of the same magnitude and opposite sign as for ordinary matter. Such opposite-statistics "ghost matter" in supersymmetric gauge theory as been considered elsewhere in a similar spirit [52-55]. So

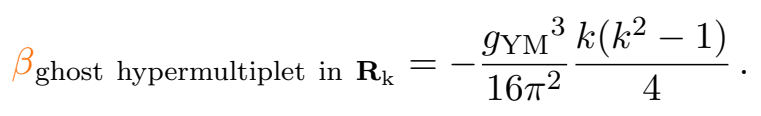

The $\mathrm{SU}(2)$ vector multiplet contribution to the $\beta$-function is

$$
\beta_{\mathrm{SU}(2) \text { vector multiplet }}=-6,
$$

so the condition for the cancellation of the $\beta$-function is

$$
\sum_{k} \frac{k\left(k^{2}-1\right)}{4} \times\left(n_{k}^{\text {(hyper) }}-n_{k}^{\text {(ghost hyper) }}\right)=+6,
$$


where $n_{k}^{\text {(hyper) }}$ and $n_{k}^{\text {(ghost hyper) }}$ are the numbers of ordinary hypermultiplets and ghost hypermultiplets, respectively, in the $k$-dimensional representation of $\mathrm{SU}(2)$.

The $\beta$-function depends only on the differences $\tilde{n}_{k}^{\text {(hyper) }} \equiv n_{k}^{\text {(hyper) }}-n_{k}^{\text {(ghost hyper) }}$, and so we can write formula (D.6) as

$$
\sum_{k} \frac{k\left(k^{2}-1\right)}{4} \times \tilde{n}_{k}^{\text {(hyper) }}=+6 .
$$

This is just the generalization of the usual $\beta$-function formula to negative numbers of hypermultiplets; the path integral with ghost hypers gives this generalized formula a concrete physical interpretation, at least in terms of a superconformal statistical system in four euclidean dimensions, if not a quantum theory in $3+1$ spacetime dimensions.

Our only intended use for this system is to serve as a nonunitary regulator for the effective vector multiplet action with various values of the $\alpha$-coefficient of the super-WZ term.

Since we only wish to define the effective theory up to the scale $\Lambda \ll|\phi|$, the nonunitary nature of the ghost hypers is irrelevant since all hypermultiplet degrees of freedom are massive at the scale set by $|\phi|$ : so long as the ghost hypers satisfy this condition, then they just serve as a nice regulator for the WZ action that has the useful property of preserving the spontaneously broken $\mathcal{N}=2$ superconformal symmetry. Similar regulators for $\mathcal{N}=4$ theories involving ghost matter have been considered elsewhere [52-55]. The present ghost regulators are similar to those of [55], which are simpler than those of [52-54], in that the latter theories considered there involved nonunitary degrees of freedom in the gauge sector as well as in the matter sector, necessarily so in order to preserve the full $\mathcal{N}=4$ supersymmetry. Our regulating theories, like those of [55], have nonunitarity only in the matter sector.

We therefore need to engineer a vacuum manifold consisting solely of an Abelian vector multiplet, with no additional massless degrees of freedom from the hypers when the vector multiplet scalar has a nonzero vev. That is, we wish to exclude the case of an "enhanced" Coulomb branch or its ghost generalization. To achieve this, it is necessary and sufficient to choose all the representations to be even-dimensional. Then the mass matrix for the hypers, $t_{\mathbf{R}}^{A} \phi^{A}$, has no vanishing eigenvalues for nonzero $\phi^{A}$, and the vacuum manifold is a pure Coulomb branch. So we will restrict our representation content to $k$ even. With this criterion, all ghost degrees of freedom have masses of order $|\phi|$ and are above the cutoff $\Lambda$.

Now let us write an expression for the $a$-anomaly of the underlying CFT. So long as the $\beta$-function vanishes, the gauge coupling $\tau$ is marginal and the anomaly is $\tau$-independent, and we can compute the Weyl anomaly accurately in free field theory. Just as for the gauge anomaly, the ghost hypermultiplets contribute to the Weyl anomaly oppositely to the ordinary hypermultiplets in the same representation. Thus we have the total $a$ - coefficient

$$
a_{\mathrm{CFT}}^{[\mathrm{AEFJ}]}=a_{\mathrm{SU}(2) \text { vector multiplet }}^{[\mathrm{AEFJ}]}+a_{\mathrm{matter}}^{[\mathrm{AEFJ}]}=\frac{5}{8}+\frac{1}{24} \times \sum_{k} k \tilde{n}_{k}^{(\mathrm{hyper})} .
$$

If we have chosen all the $k$ to be even, then there are no massless degrees of freedom on the Coulomb branch other than the vector multiplet, and so the Coulomb branch EFT has

$$
a_{\mathrm{EFT}}^{[\mathrm{AEF}]}=a_{\mathrm{U}(1) \text { vector multiplet }}^{[\mathrm{AEFJ}]}=+\frac{5}{24} .
$$


Then the anomaly mismatch in AEFJ units is

$$
(\Delta a)^{[\mathrm{AEFJ}]} \equiv a_{\mathrm{EFT}}^{[\mathrm{AEFJ}]}-a_{\mathrm{EFT}}^{[\mathrm{AEFJ}]}=\frac{5}{12}+\frac{1}{24} \times \sum_{k} k \tilde{n}_{k}^{(\mathrm{hyper})}
$$

and the $\alpha$-coefficient then comes out to

$$
\alpha \equiv 2 \times(\Delta a)^{[\mathrm{AEFJ}]}=\frac{5}{6}+\frac{1}{12} \sum_{k} k \tilde{n}_{k}^{\text {(hyper) }} .
$$

We include only even $k$ in the sum, but other than that there is no restriction on the $\tilde{n}_{k}$ other than the requirement (D.7) that the $\beta$-function vanishes.

Conformal combinations of matter and ghost matter. Since the $\tilde{n}_{k}$ can be positive or negative, there are many ways to satisfy equation (D.7) while giving different values for $\alpha$ as determined by equation (D.11). For instance, for $\tilde{n}_{4}^{\text {(hyper) }}$ any integer, we can take

$$
\begin{cases}\tilde{n}_{2}^{\text {(hyper })}=4-10 \tilde{n}_{4}^{\text {(hyper) }}, & \\ \tilde{n}_{k}=0 & \forall k \neq 2,4 .\end{cases}
$$

Then the $\beta$-function cancellation equation (D.7) is satisfied, and the value of $\alpha$ is

$$
\alpha=\frac{3}{2}-\frac{4}{3} \tilde{n}_{4}^{\text {(hyper) }} .
$$

Super-Weyl invariance of the ghost-hyper theories. The vanishing of the $\beta$-function means that these theories are scale-invariant. However we can see that they are not only scale-invariant, they are Weyl-invariant on curved space and therefore super-Weylinvariant on curved superspace [56, 57].

The action for ghost hypers is Weyl-invariant at the Lagrangian level exactly as it is for ordinary hypers: for both types of multiplet, the action is exactly quadratic in hypermultiplet degrees of freedom, and the ghost hypers are taken to have exactly the same super-Weyl transformation laws as the ordinary hypers. So even though nonunitary scale-invariant theories are not Weyl-invariant in general, the ghost-hyper SCFTs are special cases which are in fact super-Weyl invariant. This is important to emphasize, because we will use super-Weyl invariance, not just scale invariance, as a symmetry to eliminate higherderivative $F$-terms in the Coulomb branch EFT of the ghost-hyper theories.

For vector multiplet actions, $\mathcal{N}=2$ super-Weyl invariance follows automatically from Weyl-invariance and $\mathcal{N}=2$ susy because the $\mathcal{N}=2$ supergravity background has a superspace formalism which couples naturally to half-superspace $F$-terms for vector multiplets as well as full-superspace terms. For hypermultiplet $F$-terms, maintaining manifest supersymmetry off-shell is more subtle, requiring more sophisticated superspace formalisms such as harmonic superspace or projective superspace, to which we know of no currently developed formalism for coupling to a curved superbackground.

However it is possible to see directly that the action for ghost hypermultiplets must be super-Weyl-invariant, if the action for ordinary hypermultiplets is super-Weyl-invariant. There are two more or less equivalent ways to see this, one "on-shell" and one "off-shell". 
Both forms of the proof use the fact that the action for hypermultiplets, both ghost type and ordinary type, is exactly quadratic in the hypermultiplet fields.

The on-shell, operator argument is as follows. Since the action is exactly quadratic in the hypermultiplet degrees of freedom, so must be the stress tensor, supersymmetry generators, and other currents. In particular, the virial current would have to be quadratic in hypermultiplet degrees of freedom, and there is no candidate virial current that is quadratic in hypermultiplet degrees of freedom.

This operator proof translates into an off-shell argument in component fields, as follows:

Given an $\mathcal{N}=2$ supergravity background and a fixed (not necessarily supersymmetric or on-shell) background for the vector multiplet, we can perform a super-Weyl transformation on the metric and vector-multiplet degrees of freedom.

The full action for vector and hypermultiplets is super-Weyl invariant, and thus for an arbitrary super-Weyl transformation of the background metric and dynamical SU(2) vector multiplet, there must exist a corresponding transformation on the components of the hypermultiplet that leaves the Lagrangian invariant, not just up to a total derivative or local susy transformation, but invariant exactly, since the virial current must vanish. The action is exactly quadratic, and the transformation of the off-shell hypermultiplet component fields under the super-Weyl transformation is linear.

The exact same super-Weyl transformation can be applied as a linear transformation to the off-shell ghost hypermultiplet component fields, and the action will necessarily still be invariant: for a quadratic action for a complex field, a linear transformation on a bosonic field leaves the action invariant if and only if the corresponding action for a fermionic field also does so: for a quadratic action for a complex field, the statistics of the field are irrelevant to the invariance of the action so long as the transformation is linear.

We therefore conclude that the fixed points with ghost-hypermultiplets are invariant under the same super-Weyl transformations as the SCFTs with ordinary unitary hypermultiplets.

\section{E Saddle point value of the classical action}

In section 4 we have given an indirect proof that the power law corrections $\hat{K}_{m} / \mathcal{J}$ to the $q_{n}$ for any one-dimensional Coulomb branch, must be given by the universal polynomials $\hat{P}_{m+1}^{\text {[univ] }}(\alpha)$ given in eq. (3.4), even in a non-Lagrangian theory with no marginal coupling and no evident reason to obey the gauge-theoretic recursion relations from which the $\hat{P}_{m+1}(\alpha)$ were derived. Our proof was somewhat abstract, and relies on the well-definiteness of unfamiliar path integrals involving nonunitary matter in the hypermultiplet sector.

Due to the absence of higher-derivative $F$-terms, the $\hat{K}_{m}$ are certainly well-defined universal, and computable within the Coulomb-branch EFT itself, independent of any data other than the $\alpha$-coefficient. It is therefore possible in principle to check our result directly by computing correlators in the EFT.

In this appendix we perform the simplest possible check of deriving the leading coefficients of the $\hat{P}_{m+1}$ polynomials, which give the terms of order $\alpha^{m+1} / \mathcal{J}^{m}$ in the expansion of $q_{n}$. 
The terms of order $\alpha^{m+1} / \mathcal{J}^{m}$, i.e., the leading terms in the polynomials $\hat{P}_{m+1}(\alpha)$, are most conveniently computed by using the BPS helical property of the classical solution: the only change in the classical helical solution, at any order in $\alpha$, is the equilibrium value of $|\phi|$ at fixed $\mathcal{J}$ and fixed frequency $\omega=1 / r$.

Modulo $\mathcal{J}$-independent normalization constants for the $S^{4}$ partition function and the operator $\mathcal{O}$ itself, the $q_{n}$ are simply given by the partition function with sources:

$$
q_{n}=\log \left[Z_{0} \mathcal{Y}_{n}\right]=\log \left(Z_{n}\right)=\exp \left\{-W_{n}\right\}
$$

where $Z_{n}$ is the path integral over the action

$$
S_{n} \equiv S_{\text {free }}+\text { super-wz }+S_{\text {sources }}
$$

The sum of tree diagrams contributing to $W_{n}$ is simply the classical action at the saddle point, including the free action, sources, and Wess-Zumino term. Therefore the full $\alpha$-dependent expression for the classical action, will give us the leading terms of all the polynomials in $\hat{P}_{m+1}$ :

$$
\left.\sum_{m} q_{n}\right|_{\alpha^{m+1} / n^{m} \text { term }}=-\left.S_{n}\right|_{\text {saddle point value }} .
$$

In the $\tau, \beta$ variables, the (Lorentz mostly-plus signature) classical action on the cylinder $S^{3} \times$ time is

$$
S^{(\text {Lorentzian })}=\int d^{4} x \sqrt{|-g|} \mathcal{L}^{\text {(Lorentzian) }},
$$

which we can decompose into the kinetic and wz term:

$$
\mathcal{L}^{(\text {Lorentzian })}=\mathcal{L}_{\text {kin }}^{(\text {Lorentzian })}+\mathcal{L}_{\text {super-WZ }}^{(\text {Lorentzian })}
$$

The kinetic term is simply

$$
\mathcal{L}_{\text {kin }}^{\text {(Lorentzian) }} \equiv-\mu \exp \{-2 \tau\}\left[(\partial \tau)^{2}+(\partial \beta)^{2}-\frac{1}{6} \operatorname{Ric}_{4}\right]
$$

where the Ricci scalar is given by $\mathrm{Ric}_{4}=\mathrm{Ric}_{3}=6 / r^{2}$.

We have written the wz term in eq. (B.21). The sources are at infinity and do not affect the helical solution at all, except insofar as they set the value $\mathcal{J}$ of the $R$-charge for the solution. The frequency of a BPS helical solution is in general fixed by the BPS property (for a realization of this fact in the contest of string theory see [58]) and indeed the EOM $\dot{\beta}^{2}=1 / r^{2}$ for $\tau$ tells us immediately that $\beta= \pm 1 / r$ is the only allowed frequency for a helical solution, since the undifferentiated $\tau$ appears only in the kinetic term $\mathcal{L}_{\text {kin }}$ and its variational equation fixes $\beta^{2}=1 / r^{2}$, with the one sign for $\dot{\beta}$ corresponding to the BPS helical solution and the other sign corresponding to an anti-BPs helical solution. 
For any given $\tau$, the $R$-charge density is simply the derivative of the Lagrangian density with respect to $\dot{\beta}$ :

$$
\begin{aligned}
\rho & =\frac{\delta \mathcal{L}}{\delta \dot{\beta}}=2 \mu^{2} \exp \{-2 \tau\} \dot{\beta}-\frac{8(\Delta a)^{[\mathrm{KS}]}}{r^{2}} \dot{\beta}-8(\Delta a)^{[\mathrm{KS}]} \dot{\beta}^{3} \\
& =\operatorname{sgn}(\dot{\beta})\left[2 \mu^{2} \exp \{-2 \tau\} r^{-1}-\frac{8(\Delta a)^{[\mathrm{KS}]}}{r^{3}}-8(\Delta a)^{[\mathrm{KS}]} r^{-3}\right] \\
& =\frac{2 \operatorname{sgn}(\dot{\beta})}{r}\left[\mu^{2} \exp \{-2 \tau\}-\frac{8(\Delta a)^{[\mathrm{KS}]}}{r^{2}}\right] \\
& =\frac{2 \operatorname{sgn}(\dot{\beta})}{r}\left[|\phi|^{2}-\frac{8(\Delta a)^{[\mathrm{KS}]}}{r^{2}}\right] \\
& =\frac{1}{\mathcal{A}_{S^{3}}} \mathcal{J}=\frac{1}{2 \pi^{2} r^{3}} \times \mathcal{J},
\end{aligned}
$$

where in the last equality we have used

$$
\mathcal{J}=\mathcal{A}_{S^{3}} \times \rho=2 \pi^{2} r^{3} \times \rho .
$$

We see that $\operatorname{sgn}(\dot{\beta})=\operatorname{sgn}(\mathcal{J})$ and $\mathcal{J} / \dot{\beta}=|\mathcal{J}|$, so

$$
\mu^{2} \exp \{-2 \tau\}-\frac{8(\Delta a)^{[\mathrm{KS}]}}{r^{2}}=|\phi|^{2}-\frac{8(\Delta a)^{[\mathrm{KS}]}}{r^{2}}=\frac{1}{4 \pi^{2} r^{2}} \times|\mathcal{J}| .
$$

Solving for $|\phi|^{2}$ gives

$$
\mu^{2} \exp \{-2 \tau\}=|\phi|^{2}=\frac{1}{4 \pi^{2} r^{2}} \times|\mathcal{J}|+\frac{8(\Delta a)^{[\mathrm{KS}]}}{r^{2}} .
$$

Using the translation between the AEFJ normalization and the AEFJ normalization in [21] for the $a$-anomaly, given in appendix A of [1] as

$$
a^{[\mathrm{KS}]}=\frac{1}{16 \pi^{2}} a^{[\mathrm{AEFJ}]}, \quad \quad a^{[\mathrm{AEFJ}]}=16 \pi^{2} a^{[\mathrm{KS}]},
$$

we have

$$
\mu^{2} \exp \{-2 \tau\}=|\phi|^{2}=\frac{1}{4 \pi^{2} r^{2}}|\mathcal{J}|+\frac{(\Delta a)^{[\mathrm{AEFJ}]}}{2 \pi^{2} r^{2}},
$$

and using the definition of the $\alpha$-coefficient,

$$
\alpha=2(\Delta a)^{[\mathrm{AEFJ}]},
$$

we have

$$
\mu^{2} \exp [-2 \tau]=|\phi|^{2}=\frac{1}{4 \pi^{2} r^{2}}|\mathcal{J}|+\frac{\alpha}{4 \pi^{2} r^{2}}=\frac{1}{4 \pi^{2}}(|\mathcal{J}|+\alpha),
$$

as an exact statement in the classical solution. Comparing the value at $\alpha=0$, we have

$$
\frac{|\phi|^{2}}{\left.|\phi|^{2}\right|_{\alpha=0}}=f^{2}(\alpha)
$$


with

$$
f(\alpha)=\sqrt{1+\frac{\alpha}{|\mathcal{J}|}}=\sqrt{1+\hat{\alpha}} .
$$

Substituting $\dot{\beta} \rightarrow \frac{1}{r}$, and solving for $\tau$ in terms of $\mathcal{J}$ yields

$$
\left|\phi_{\alpha}\right|^{2}=\frac{1}{4 \pi^{2} r^{2}} \times|\mathcal{J}|+\frac{\alpha}{4 \pi^{2} r^{2}}=\frac{1}{4 \pi^{2}}(|\mathcal{J}|+\alpha)=f^{2}(\alpha)\left|\phi_{0}\right|^{2},
$$

where $\phi_{\alpha}$ is the solution for nonzero $\alpha$ and $\phi_{0}$ is the solution at $\alpha=0$.

The ratio $|\phi| /|\phi|_{0}$ is Weyl-invariant and is also constant over space (equal to $f(\alpha)$ ) in cylinder frame; therefore it is constant in all conformal frames.

Modulo $\phi$-independent terms proportional to $n^{1}$, the saddle-point value of the source term in the action is

$$
\begin{aligned}
S_{\text {source }}[\alpha] & =-\mathcal{J} \log \left[\phi_{\alpha}(x)\right]-\mathcal{J} \log \left[\bar{\phi}_{\alpha}(y)\right] \\
& =S_{\text {source }}[\alpha=0]-\mathcal{J} \log \left[f^{2}(\alpha)\right] \\
& =S_{\text {source }}[\hat{\alpha}=0]-\mathcal{J} \log [1+\hat{\alpha}] .
\end{aligned}
$$

The kinetic term is $R$-symmetry invariant and exactly quadratic in $|\phi|$. In [1] it was found that

$$
|\partial \phi(x)|^{2}=\frac{\mathcal{J}}{2} \delta^{(4)}\left(x-x_{1}\right)+\frac{\mathcal{J}}{2} \delta^{(4)}\left(x-x_{2}\right)
$$

so the saddle-point value of the free kinetic term on flat space, including $\delta$-function contributions at the insertion points, is

$$
\begin{aligned}
S_{\text {kinetic }}[\alpha] & =f(\alpha)^{2} S_{\text {kinetic }}[\alpha=0]=(1+\hat{\alpha}) S_{\text {kinetic }}[\alpha=0] \\
& =(1+\hat{\alpha}) S_{\text {kinetic }}[\hat{\alpha}=0]=\mathcal{J}(1+\hat{\alpha}) .
\end{aligned}
$$

Finally, the super-wz term contains only gradients of $\beta$ and differentiated logarithms of $|\bar{\phi}|$, and therefore $f(\alpha)$ drops out of the super-Wz term altogether, except for the Eulerdensity piece:

$$
\begin{aligned}
S_{\text {super-WZ }}^{(\text {Euclidean })}[\alpha]= & \left.S_{\text {super-WZ }}^{(\text {Euclidean })}\right|_{\phi=\phi_{\alpha}}=\left.S_{\text {super-WZ }}^{\text {Euclidean })}\right|_{\phi=\phi_{0}}-\alpha \log (f(\alpha)) \int \mathrm{d}^{4} x \sqrt{-g} E_{4}^{\mathbb{Z}} \\
= & \left(\operatorname{order} \alpha^{1} \log (\mathcal{J}) \text { term }\right)+\left(\text { order } \alpha^{1} \mathcal{J}^{0} \text { term }\right) \\
& -\alpha \log (f(\alpha)) \int \mathrm{d}^{4} x \sqrt{-g} E_{4}^{\mathbb{Z}} .
\end{aligned}
$$

Of these, the first was already computed in [1] and is equal to $-\alpha \log (\mathcal{J})$ and thus contributes to $q_{n}$ as $+\alpha \log (\mathcal{J})$. For purposes of the computation in this section we are really only interested in terms that are order $\hat{\alpha}^{2}$ and higher, and so we ignore both the first two terms in the wz action. These two contain only terms linear in $\alpha$; only the third term contains terms of order $\alpha^{2}$ and larger.

As in [1] we convert the KS normalization of the Euler density into the integernormalization of the Euler density, i.e. the one normalized so that

$$
\int d^{4} x \sqrt{|g|} E_{4}^{\mathbb{Z}}=\chi\left(\mathbf{X}_{4}\right) \in \mathbb{Z}
$$


with the convention $\chi\left(S^{4}\right)=2$. The proportionality constant is [1]

$$
E_{4}^{[\mathrm{KS}]}=32 \pi^{2} E_{4}^{\mathbb{Z}}, \quad E_{4}^{\mathbb{Z}}=\frac{1}{32 \pi^{2}} E_{4}^{[\mathrm{KS}]}
$$

so the third term of (E.21) is

$$
\begin{aligned}
\left.\mathcal{L}_{\text {super-WZ }}^{(\text {Euclidean })}[\alpha]\right|_{E_{4} \log [f(\alpha)]} & =(\Delta a)^{[\mathrm{KS}]} \tau E_{4}^{[\mathrm{KS}]}=\frac{1}{16 \pi^{2}}(\Delta a)^{[\mathrm{AEFJ}]} \times\left(32 \pi^{2}\right) \tau E_{4}^{[\mathrm{Z}]} \\
& =2 \times(\Delta a)^{[\mathrm{AEFJ}]} \times \tau E_{4}^{[\mathbb{Z}]}=\alpha \times \tau E_{4}^{[\mathbb{Z}]}
\end{aligned}
$$

so for the four-sphere

$$
\begin{aligned}
\left.S_{\text {super-WZ }}^{(\text {Euclidean }}[\alpha]\right|_{E_{4} \log [f(\alpha)]} & 2 \alpha \tau=-2 \alpha \log (|\phi| / \mu)=-\alpha \times \log \left(|\phi|^{2} / \mu^{2}\right) \\
= & -\mathcal{J} \hat{\alpha} \times \log \left(|\phi|^{2} / \mu^{2}\right) \\
= & -\mathcal{J} \hat{\alpha} \times \log \left(\left|\phi_{0}\right|^{2} / \mu^{2}\right)-\mathcal{J} \hat{\alpha} \times \log \left(\left|\phi_{a}\right|^{2} /\left|\phi_{0}\right|^{2}\right) \\
= & -\mathcal{J} \hat{\alpha} \times \log \left(\left|\phi_{0}\right|^{2} / \mu^{2}\right)-\mathcal{J} \hat{\alpha} \times \log \left[f^{2}(\alpha)\right] \\
& -\mathcal{J} \hat{\alpha} \times \log \left(\left|\phi_{0}\right|^{2} / \mu^{2}\right)-\mathcal{J} \hat{\alpha} \times \log [1+\hat{\alpha}] .
\end{aligned}
$$

We can write this as

$$
\begin{aligned}
\left.S_{\text {super-WZ }}^{(\text {Euclidean })}[\alpha]\right|_{E_{4} \log [f(\alpha)]} & =-\mathcal{J} \hat{\alpha} \times \log \left(\left|\phi_{0}\right|^{2} / \mu^{2}\right)-\mathcal{J} \hat{\alpha} \times \log [1+\hat{\alpha}] \\
& =\left(\operatorname{order} \mathcal{J}^{1} \hat{\alpha}^{1}\right)-\mathcal{J} \hat{\alpha} \times \log [1+\hat{\alpha}] .
\end{aligned}
$$

So putting it all together, we have

$$
\begin{aligned}
& S_{\text {kinetic }}^{\text {Euclican }}[\alpha]=(\text { affine in } \alpha), \\
& S_{\text {source }}^{\text {(Euclidean })}[\alpha]=\left(\operatorname{order} \mathcal{J}^{1} \hat{\alpha}^{0}\right)-\mathcal{J} \log (1+\hat{\alpha}), \\
& S_{\text {super-WZ }}^{\text {Euclidean }}[\alpha]=(\text { affine in } \alpha)-\mathcal{J} \hat{\alpha} \log (1+\hat{\alpha}),
\end{aligned}
$$

and the complete saddle point action is

$$
\begin{aligned}
S_{\text {saddle point, total }}^{(\text {Euclidean }}[\alpha] & =(\text { affine in } \alpha)-\mathcal{J}(1+\hat{\alpha}) \log (1+\hat{\alpha}) \\
& =(\text { affine in } \alpha)-\mathcal{J} \times\left[\alpha+\sum_{m \geq 1}(-1)^{m+1} \frac{\hat{\alpha}^{m+1}}{m(m+1)}\right] \\
& =(\text { affine in } \alpha)-\mathcal{J} \times\left[\sum_{m \geq 1}(-1)^{m+1} \frac{\hat{\alpha}^{m+1}}{m(m+1)}\right]
\end{aligned}
$$


so for all $m \geq 1$ we have

$$
\begin{aligned}
\left.K_{n} n^{-m}\right|_{\text {order } \alpha^{m+1}} & =\left.\hat{K}_{m} \mathcal{J}^{-m}\right|_{\text {order } \hat{\alpha}^{m+1}}=\left.\mathcal{J}^{+1} \hat{P}_{m+1}(\hat{\alpha})\right|_{\text {order } \hat{\alpha}^{m+1}} \\
& =\left.q_{n}\right|_{\text {order } \hat{\alpha}^{m+1} \mathcal{J}^{+1}}=-\left.S_{n, \text { saddle point, total }}^{\text {(Euclidean })}[\alpha]\right|_{\text {order } \hat{\alpha}^{m+1} \mathcal{J}^{+1}} \\
& =+\mathcal{J} \times(-1)^{m+1} \frac{\hat{\alpha}^{m+1}}{m(m+1)}, \quad \forall m \geq 1 .
\end{aligned}
$$

This result is to be compared with the formula for the polynomials $\hat{K}_{m}=\hat{P}_{m+1}(\alpha)$ in eq. (3.4) and gives an infinite number of direct computations consistent with our universal formula. It is a direct calculation in the EFT and it is independent of the ghost-hyper argument and independent of any UV-completion of the EFT. This infinite number of agreeing coefficients supports our argument that our formula for the power-law corrections $\hat{K}_{m}^{[\text {univ] }}$ is universal among all theories with a given value of $\alpha$, including non-Lagrangian theories.

\section{F Numerics}

In the case of $\mathcal{N}=2 \mathrm{SQCD}$ with 4 flavors the correlators that we discuss can be computed via localization [15]. The function $G_{2 n}$ is the ratio of two determinants:

$$
G_{2 n}=4^{2 n} \frac{\operatorname{det}\left(M_{n}\right)}{\operatorname{det}\left(M_{n-1}\right)},
$$

where $M_{n}$ is the upper-left $(n-1) \times(n-1)$ submatrix of the (normalized) matrix of derivatives $M$ of the partition function $Z_{0}$ :

$$
\left.M\right|_{m, n}=\frac{1}{Z_{0}} \partial^{n} \bar{\partial}^{m} Z_{0}
$$

The partition function for $\mathcal{N}=2 \mathrm{SQCD}$ is written in terms of the Barnes $G$-function [59]:

$$
Z_{0}=Z_{S^{4}}^{\mathrm{SQCD}}(\tau, \bar{\tau})=\int_{-\infty}^{\infty} \mathrm{d} a a^{2} e^{-4 a \operatorname{Im} \tau} \frac{|G(1+2 i a)|^{4}}{|G(1+i a)|^{16}}\left|Z_{\text {inst }}(i a, \tau)\right|^{2},
$$

where $Z_{\text {inst }}$ is the instanton partition function [60,61]:

$$
Z_{\text {inst }}(a, \tau)=1+\frac{1}{2}\left(a^{2}-3\right) e^{2 \pi i \tau}+\mathcal{O}\left(e^{4 \pi i \tau}\right) .
$$

For simplicity we will consider the regime $\operatorname{Im} \tau>1$ and ignore the instanton corrections. Note that in this approximation the partition function $Z_{\text {inst }}(a, \tau)$ is independent of $\operatorname{Re}(\tau)$.

Since we want to evolve the recursion relations numerically starting from an approximate initial condition for the $S^{4}$ partition function, we need to estimate the sensitivity of large- $\mathcal{J}$ correlation functions to imprecise initial conditions.

We may wish to start at some initial value $n_{i}$ greater than 0 . The recursion relation is second order, so in order to define initial conditions, we need to define both $q_{n_{i}}$ and $q_{n_{i}+1}$. 
These initial conditions are of course functions of $\tau$ and $\bar{\tau}$, but we will suppress in this section the dependence on the arguments $\tau, \bar{\tau}$ in our notation.

It is useful to write the rank-one recursion relations in their "deterministic" form. Given any initial conditions at $n_{i}, n_{i}+1$, there is always a unique solution to the recursion relations for $n \geq n_{i}+2$. One can consider two nearby solutions, separated by a small amount $\delta_{n}$, and analyze how the linearized deviation propagates to larger values of $n$. The deviation propagation equation is:

$$
\begin{aligned}
E_{n+2} & =E_{n+1} \partial_{\tau} \partial_{\bar{\tau}}\left[\log \left(E_{n+1}\right)\right]+\frac{E_{n+1}^{2}}{E_{n}}, \\
E_{n} & \equiv \exp \left\{q_{n}\right\} .
\end{aligned}
$$

In terms of $q_{n}$ itself this is

$$
\begin{aligned}
q_{n+2} & =\log \left(E_{n+2}\right)=\log \left[\exp \left\{2 q_{n+1}-q_{n}\right\}+\exp \left\{q_{n+1}\right\} \partial_{\tau} \partial_{\bar{\tau}} q_{n+1}\right] \\
& =2 q_{n+1}-q_{n}+\log \left[1+\exp \left\{q_{n}-q_{n+1}\right\} \partial_{\tau} \partial_{\bar{\tau}} q_{n+1}\right]
\end{aligned}
$$

which we can write as

$$
q_{n+2}-2 q_{n+1}+q_{n}=\log \left[1+\exp \left\{q_{n}-q_{n+1}\right\} \partial_{\tau} \partial_{\bar{\tau}} q_{n+1}\right]
$$

General evolution equation for small perturbations. Now consider some small perturbations $\delta_{n_{i}}, \delta_{n_{i}+1}$ of the initial conditions that are small relative to the initial conditions $q_{n_{i}}, q_{n_{i}+1}$ themselves:

$$
r_{n_{i}}, r_{n_{i}+1} \ll 1, \quad r_{n} \equiv \frac{\delta_{n}}{q_{n}} .
$$

Within this assumption, the propagation equation for $r_{n}$ can always be treated linearly.

Suppose we have an unperturbed solution to the recursion relations (F.6), for $n \geq n_{i}+2$, and we perturb it with some small change to the initial conditions $\delta_{n_{i}}, \delta_{n_{i}}$, so

$$
\begin{aligned}
& q_{n_{i}}^{\text {(new) }}=q_{n_{i}}^{\text {(unp) }}+\delta_{n_{i}}, \\
& q_{n_{i}+1}^{\text {(new) }}=q_{n_{i}+1}^{\text {(unp) }}+\delta_{n_{i}+1},
\end{aligned}
$$

with $\delta_{n_{i}}, \delta_{n_{i}+1}$ satisfying (F.8).

The recursion relation is of course continuous as a function of its initial conditions, so the smallness of the perturbation of the initial conditions (F.9) implies the smallness of the perturbation (F.8) for $n-n_{i}$ not too large. 
If both $q_{n}^{\text {(new) }}$ and $q_{n}^{\text {(unp) }}$ obey the recursion relations exactly for $n \geq n_{i}+2$, then the recursion relation for $\delta_{n}$ is

$$
\begin{aligned}
& \delta_{n+2}-2 \delta_{n+1}+\delta_{n} \\
& =\left[1+\frac{E_{n}^{\text {(unp })}}{E_{n+1}^{\text {(unp) }}} \partial_{\tau} \partial_{\bar{\tau}} q_{n+1}^{\text {(unp) }}\right]^{-1} \times\left(\frac{E_{n}^{\text {(unp })}}{E_{n+1}^{\text {(unp) }}} \partial_{\tau} \partial_{\bar{\tau}} \delta_{n+1}+\frac{E_{n}^{\text {(unp })}}{E_{n+1}^{\text {(unp })}} \times\left(\delta_{n}-\delta_{n+1}\right) \partial_{\tau} \partial_{\bar{\tau}} q_{n+1}^{\text {(unp })}\right)+O\left(\delta^{2}\right) \\
& =\left[1+\frac{E_{n}^{(\text {unp })}}{E_{n+1}^{\text {(unp) }}} \partial_{\tau} \partial_{\bar{\tau}} q_{n+1}^{\text {(unp) }}\right]^{-1} \times \frac{E_{n}^{\text {(unp })}}{E_{n+1}^{\text {(unp) }}} \times\left(\partial_{\tau} \partial_{\bar{\tau}} \delta_{n+1}+\left(\delta_{n}-\delta_{n+1}\right) \partial_{\tau} \partial_{\bar{\tau}} q_{n+1}^{\text {(unp })}\right)+O\left(\delta^{2}\right) \\
& =\left[\frac{E_{n+1}^{\text {(unp })}}{E_{n}^{(\text {unp })}}+\partial_{\tau} \partial_{\bar{\tau}} q_{n+1}^{\text {(unp) }}\right]^{-1} \times\left(\partial_{\tau} \partial_{\bar{\tau}} \delta_{n+1}+\left(\delta_{n}-\delta_{n+1}\right) \partial_{\tau} \partial_{\bar{\tau}} q_{n+1}^{(\text {unp })}\right)+O\left(\delta^{2}\right), \quad \forall n \geq n_{i} .
\end{aligned}
$$

Since we will be always assuming the small-deviation condition $\circ \circ \circ$, we will discard the $O\left(\delta^{2}\right)$ term, in which case we can ignore the distinction between $q^{(\text {unp })}$ and $q^{\text {(new) }}$ in the perturbation evolution equation (F.10). So treating $\delta$ as a purely infinitesimal quantity we can write:

$$
\delta_{n+2}-2 \delta_{n+1}+\delta_{n}=\left[\frac{E_{n+1}}{E_{n}}+\partial_{\tau} \partial_{\bar{\tau}} q_{n+1}\right]^{-1} \times\left(\partial_{\tau} \partial_{\bar{\tau}} \delta_{n+1}+\left(\delta_{n}-\delta_{n+1}\right) \partial_{\tau} \partial_{\bar{\tau}} q_{n+1}\right), \quad \forall n \geq n_{i} .
$$

We shall explore the question of the rate of growth of $\delta_{n}$ for different regimes of $n, \tau$, and so forth, always taking the perturbation to obey (F.8).

Evolution of perturbations of solutions with EFT asymptotics at large $\boldsymbol{n}$. Now let us examine a solution to the recursion equations in which the $q_{n_{i}}^{(\text {unp })}$ asymptotes to a solution with the large- $n$ asymptotics considered here, i.e.,

$$
q_{n_{i}}^{(\mathrm{unp})}=q_{n_{i}}^{(\mathrm{EFT})}+\mathcal{W}_{n}(\tau, \bar{\tau}),
$$

where we have defined

$$
\begin{aligned}
q_{n}^{(\mathrm{EFT})} & \equiv \mathbf{A}(\tau, \bar{\tau}) n+\mathbf{B}(\tau, \bar{\tau})+\log [\Gamma(2 n+\alpha+1)], \\
\mathcal{W}_{n}(\tau, \bar{\tau}) & =(\text { exponentially small in } n),
\end{aligned}
$$

with

$$
\begin{aligned}
& \mathbf{A}(\tau, \bar{\tau})=-2 \log [\operatorname{Im}(\tau)]-4 \log (2), \\
& \mathbf{B}(\tau, \bar{\tau})=-\left(\alpha+\frac{1}{2}\right) \log [\operatorname{Im}(\tau)]+k_{0},
\end{aligned}
$$

with $k_{0}$ independent of $\tau$ and $\bar{\tau}$.

For these asymptotics, the error propagation equation becomes

$$
\delta_{n+2}+\delta_{n}-2 \delta_{n+1}=\circ \circ \circ+(\text { exponentially small in } n) .
$$


Now we observe, as earlier in the paper, that the form (F.13), (F.14) with $\mathcal{W}_{n}=0$ identically, is an exact solution to the recursion relations for $n \geq(\circ \circ \circ)$. This is equivalent to setting

$$
q_{n}^{(\mathrm{unp})} \rightarrow q_{n}^{(\mathrm{EFT})}, \quad \mathcal{W}_{n}(\tau, \bar{\tau}) \rightarrow 0
$$

identically as the definition of the unperturbed solution, and absorbing $\mathcal{W}_{n}(\tau, \bar{\tau})$ into the definition of the deviation $\delta_{n}$,

$$
\delta_{n} \rightarrow \delta_{n}+\mathcal{W}_{n}
$$

which is consistent long as we are at $n$ which is large enough that $\mathcal{W}_{n}(\tau, \bar{\tau})$ can be treated as small. Then we have

$$
\begin{aligned}
E_{n} & =\left[\exp \left\{\mathbf{A}_{n}\right\}\right]^{n} \times[\exp \{\mathbf{B}\}] \times \Gamma(2 n+\alpha+1) \\
& =[\operatorname{Im}(\tau)]^{-2 n-\alpha-\frac{1}{2}} \times 2^{-4 n} \times \exp \left\{k_{0}\right\} \times \Gamma(2 n+\alpha+1) \\
\frac{E_{n+1}}{E_{n}} & =\frac{1}{16[\operatorname{Im}(\tau)]^{2}} \frac{\Gamma(2 n+\alpha+3)}{\Gamma(2 n+\alpha+1)}=\frac{1}{16[\operatorname{Im}(\tau)]^{2}} \frac{(2 n+\alpha+2) !}{(2 n+\alpha) !} \\
& =\frac{(2 n+\alpha+2)(2 n+\alpha+1)}{16[\operatorname{Im}(\tau)]^{2}} \\
\frac{E_{n}}{E_{n-1}} & =\frac{(2 n+\alpha)(2 n+\alpha-1)}{16[\operatorname{Im}(\tau)]^{2}} \\
\partial_{\tau} \partial_{\bar{\tau}} q_{n} & =-\left(2 n+\alpha+\frac{1}{2}\right) \partial_{\tau} \partial_{\bar{\tau}}(\log [\operatorname{Im}(\tau)])=-\left(2 n+\alpha+\frac{1}{2}\right) \times(\tau-\bar{\tau})^{-2} \\
& =-\left(2 n+\alpha+\frac{1}{2}\right) \times(2 i[\operatorname{Im}(\tau)])^{-2}=+\frac{1}{4}\left(2 n+\alpha+\frac{1}{2}\right)[\operatorname{Im}(\tau)]^{-2} \\
\partial_{\tau} \partial_{\bar{\tau}} q_{n+1} & =+\frac{1}{4}\left(2 n+\alpha+\frac{5}{2}\right)[\operatorname{Im}(\tau)]^{-2},
\end{aligned}
$$

and the propagation equation for the deviation becomes exactly

$$
\begin{aligned}
\delta_{n+2}+\delta_{n}-2 \delta_{n+1}=\frac{16(\operatorname{Im}(\tau))^{2}}{(2 n+3+\alpha)(2 n+4+\alpha)} \partial_{\tau} \partial_{\bar{\tau}} \delta_{n+1} & \\
& -\frac{2(4 n+2 \alpha+5)}{(2 n+\alpha+3)(2 n+\alpha+4)}\left(\delta_{n+1}-\delta_{n}\right) .
\end{aligned}
$$

Even at the linearized level, this equation is nontrivial, and depends on the decomposition of the error into eigenvalues of the Laplacian on the upper half plane or its quotient under the modular group. We do not analyze the propagation of errors for general perturbations. Instead, we use the fact that the perturbative piece of $Z_{0}(\tau, \bar{\tau})$ is a good approximation at weak coupling. As pointed out in [2], the clash between weak coupling and large $\mathcal{J}$ can be avoided if one considers the limit $\mathcal{J} \rightarrow \infty$ while taking $\lambda \equiv 2 \pi \mathcal{J} / \operatorname{Im}(\tau)$ fixed. Since our formula for the power-law corrections is $\tau$-independent for rank-one theories, these two limits coincide for the power-law piece $\log (\Gamma(\mathcal{J}+\alpha+1))$, differing only in the behavior of the nonuniversal exponential correction. We can therefore isolate this correction easily in 
the fixed- $\lambda$ limit, in which the instanton contributions to $Z_{0}(\tau, \bar{\tau})$ go to zero exponentially in $n$.

One might expect the exponentially small corrections to be associated with the breakdown of the EFT altogether, capturing the leading effects of massive states propagating over distances on the infrared scale, as discussed in section 2.5. One would therefore anticipate exponentially small corrections proportional to $\propto \exp \left[-\kappa \lambda^{1 / 2}\right]$, with $\kappa$ some fixed number depending on the geometry of the virtual propagation, but not on $n$ or $\tau, \bar{\tau}$. Numerically, we find a remarkably accurate match to such an exponential, with $\kappa=\sqrt{\pi} / 2$, as shown below.

In section 2.3 we have seen that only the coefficients of $n^{0}$ and $n^{1}$ in the asymptotic expansion of $q_{n}(\tau, \bar{\tau})$ are expected to depend on $\tau$. This means that the second variation in $n$ of $q_{n}(\tau, \bar{\tau})$ is $\tau$-independent. Let $\triangle$ be the difference operator $\Delta_{n} q_{n}=q_{n+1}-q_{n}$. We want to compute the second difference

$$
\triangle_{n}^{2} q_{n}^{(\text {loc })}(\tau, \bar{\tau})=q_{n+2}^{\text {(loc })}(\tau, \bar{\tau})-2 q_{n+1}^{\text {(loc })}(\tau, \bar{\tau})+q_{n}^{(\text {loc })}(\tau, \bar{\tau})
$$

and compare it with the result in eq. (A.22):

$$
\triangle_{n}^{2} q_{n}^{\mathrm{EFT}}=\log \left(\frac{(2 n+\alpha+3)(2 n+\alpha+4)}{(2 n+\alpha+1)(2 n+\alpha+2)}\right) .
$$

Figure 1 shows the results of a numerical computation for imaginary values of $\tau$ between 1 and 60 and for $n$ between 1 and 40, representing the values of $\triangle_{n}^{2} q^{(\text {loc })}$ as function of $\tau$ at fixed values of $n$. We see that quite rapidly, already for $\tau \simeq 4 i$, the $\tau$-dependence drops for all values of $n$. The asymptotic value is well approximated by $\triangle_{n}^{2} q_{n}^{\mathrm{EFT}}$ for $n$ larger that $n \gtrsim 5$, where the discrepancy is of order $1-\triangle_{n}^{2} q^{\mathrm{EFT}} /\left.\triangle_{n}^{2} q^{(\mathrm{loc})}\right|_{n=5, \tau \lesssim 1} \approx 1 \%$. At $n=1$, the discrepancy is of order $1-\triangle_{n}^{2} q^{\mathrm{EFT}} /\left.\triangle_{n}^{2} q^{(\mathrm{loc})}\right|_{n=1, \tau \lesssim 1} \approx 8 \%$.

The numerical data can help us estimate the $\tau$ and $n$ dependence of the difference $\triangle_{n}^{2}\left(q_{n}^{\text {loc }}-q_{n}^{\text {us }}\right)$. As discussed in section 2.5 , we expect the leading contribution to the difference to have the form

$$
q_{n}^{(\mathrm{loc})}-q_{n}^{\mathrm{EFT}} \sim f_{n}(\tau, \bar{\tau}) e^{-\kappa \sqrt{n / \operatorname{Im} \tau}}=f_{n}(\tau, \bar{\tau}) e^{-\kappa \sqrt{\lambda /(4 \pi)}},
$$

where $\lambda=n g^{2}=4 \pi n / \operatorname{Im} \tau$. To verify this conjecture and estimate the proportionality factor $f_{n}(\tau, \bar{\tau})$ and the coefficient $\kappa$ we have computed the difference as a function of $\tau$, keeping the ratio $n / \operatorname{Im} \tau=\lambda /(4 \pi)$ fixed (see figure 2). The numerical data is consistent with $f_{n}(\tau, \bar{\tau})$ being a constant approximately equal to $f_{n}(\tau, \bar{\tau}) \approx 1.6$ and $\kappa \approx \pi$. Already for $\tau \approx 3$ our conjecture seems to reproduce the localization data to high accuracy. Interestingly, this single exponential term to our solution $q_{n}^{(u s)}(\tau, \bar{\tau})$ seems to account for the discrepancy $\triangle_{n}^{2}\left(q_{n}^{\text {(loc) }}-q_{n}^{\mathrm{EFT}}\right)$ both in the small- $\tau$, large- $n$ (i.e. large- $\lambda$ ) regime and in the large- $\tau$ regime (see figure 3 ).

Open Access. This article is distributed under the terms of the Creative Commons Attribution License (CC-BY 4.0), which permits any use, distribution and reproduction in any medium, provided the original author(s) and source are credited. 


\section{References}

[1] S. Hellerman and S. Maeda, On the Large $R$-charge Expansion in $\mathcal{N}=2$ Superconformal Field Theories, JHEP 12 (2017) 135 [arXiv:1710.07336] [INSPIRE].

[2] A. Bourget, D. Rodriguez-Gomez and J.G. Russo, A limit for large $R$-charge correlators in $\mathcal{N}=2$ theories, JHEP 05 (2018) 074 [arXiv: 1803.00580] [INSPIRE].

[3] S. Hellerman, D. Orlando, S. Reffert and M. Watanabe, On the CFT Operator Spectrum at Large Global Charge, JHEP 12 (2015) 071 [arXiv: 1505.01537] [INSPIRE].

[4] L. Álvarez-Gaumé, O. Loukas, D. Orlando and S. Reffert, Compensating strong coupling with large charge, JHEP 04 (2017) 059 [arXiv: 1610.04495] [INSPIRE].

[5] O. Loukas, D. Orlando and S. Reffert, Matrix models at large charge, JHEP 10 (2017) 085 [arXiv: 1707.00710] [INSPIRE].

[6] O. Loukas, A matrix CFT at multiple large charges, JHEP 06 (2018) 164 [arXiv: 1711.07990] [INSPIRE].

[7] S. Hellerman, S. Maeda and M. Watanabe, Operator Dimensions from Moduli, JHEP 10 (2017) 089 [arXiv:1706.05743] [INSPIRE].

[8] D. Banerjee, S. Chandrasekharan and D. Orlando, Conformal dimensions via large charge expansion, Phys. Rev. Lett. 120 (2018) 061603 [arXiv:1707.00711] [InSPIRE].

[9] R. Rattazzi, V.S. Rychkov, E. Tonni and A. Vichi, Bounding scalar operator dimensions in $4 D$ CFT, JHEP 12 (2008) 031 [arXiv:0807.0004] [INSPIRE].

[10] S. El-Showk, M.F. Paulos, D. Poland, S. Rychkov, D. Simmons-Duffin and A. Vichi, Solving the $3 d$ Ising Model with the Conformal Bootstrap II. c-Minimization and Precise Critical Exponents, J. Stat. Phys. 157 (2014) 869 [arXiv:1403.4545] [InSPIRE].

[11] S. El-Showk, M.F. Paulos, D. Poland, S. Rychkov, D. Simmons-Duffin and A. Vichi, Solving the 3D Ising Model with the Conformal Bootstrap, Phys. Rev. D 86 (2012) 025022 [arXiv: 1203.6064] [INSPIRE].

[12] S. Rychkov, EPFL Lectures on Conformal Field Theory in D $\geq 3$ Dimensions, SpringerBriefs in Physics Series, Springer, Cham Switzerland (2016) [arXiv: 1601.05000] [INSPIRE].

[13] D. Simmons-Duffin, The Conformal Bootstrap, in proceedings of the Theoretical Advanced Study Institute in Elementary Particle Physics: New Frontiers in Fields and Strings (TASI 2015), Boulder, CO, U.S.A., 1-26 June 2015, pp. 1-74 [arXiv: 1602.07982] [InSPIRE].

[14] L.F. Alday, Large Spin Perturbation Theory for Conformal Field Theories, Phys. Rev. Lett. 119 (2017) 111601 [arXiv:1611.01500] [INSPIRE].

[15] E. Gerchkovitz, J. Gomis, N. Ishtiaque, A. Karasik, Z. Komargodski and S.S. Pufu, Correlation Functions of Coulomb Branch Operators, JHEP 01 (2017) 103 [arXiv: 1602.05971] [INSPIRE].

[16] M. Baggio, V. Niarchos and K. Papadodimas, tt* equations, localization and exact chiral rings in $4 d \mathcal{N}=2$ SCFTs, JHEP 02 (2015) 122 [arXiv: 1409.4212] [INSPIRE].

[17] M. Baggio, V. Niarchos and K. Papadodimas, Exact correlation functions in $\mathrm{SU}(2) \mathcal{N}=2$ superconformal QCD, Phys. Rev. Lett. 113 (2014) 251601 [arXiv:1409.4217] [INSPIRE].

[18] M. Baggio, V. Niarchos and K. Papadodimas, On exact correlation functions in $\mathrm{SU}(N)$ $\mathcal{N}=2$ superconformal QCD, JHEP 11 (2015) 198 [arXiv:1508.03077] [INSPIRE]. 
[19] P.C. Argyres and M. Martone, Scaling dimensions of Coulomb branch operators of $4 d \mathrm{~N}=2$ superconformal field theories, arXiv:1801.06554 [INSPIRE].

[20] M. Caorsi and S. Cecotti, Geometric classification of 4d $\mathcal{N}=2$ SCFTs, JHEP 07 (2018) 138 [arXiv: 1801.04542] [INSPIRE].

[21] D. Anselmi, D.Z. Freedman, M.T. Grisaru and A.A. Johansen, Nonperturbative formulas for central functions of supersymmetric gauge theories, Nucl. Phys. B 526 (1998) 543 [hep-th/9708042] [INSPIRE].

[22] Z. Komargodski and A. Schwimmer, On Renormalization Group Flows in Four Dimensions, JHEP 12 (2011) 099 [arXiv: 1107.3987] [InSPIRE].

[23] A. Schwimmer and S. Theisen, Spontaneous Breaking of Conformal Invariance and Trace Anomaly Matching, Nucl. Phys. B 847 (2011) 590 [arXiv:1011.0696] [InSPIRE].

[24] N. Bobev, H. Elvang and T.M. Olson, Dilaton effective action with $N=1$ supersymmetry, JHEP 04 (2014) 157 [arXiv: 1312.2925] [INSPIRE].

[25] P.C. Argyres, A.M. Awad, G.A. Braun and F.P. Esposito, Higher derivative terms in $N=2$ supersymmetric effective actions, JHEP 07 (2003) 060 [hep-th/0306118] [INSPIRE].

[26] P.C. Argyres, A.M. Awad, G.A. Braun and F.P. Esposito, Higher derivative terms in $N=2$ SUSY effective actions, in proceedings of the 3rd International Symposium on Quantum Theory and Symmetries (QTS-3), Cincinnati, Ohio, U.S.A., 10-14 September 2003, pp. 287-293 [hep-th/0402203] [INSPIRE].

[27] P.C. Argyres, A.M. Awad, G.A. Braun and F.P. Esposito, On superspace Chern-Simons-like terms, JHEP 02 (2005) 006 [hep-th/0411081] [INSPIRE].

[28] P.C. Argyres, A. Awad, P. Moomaw and J. Wittig, Holomorphic higher-derivative terms in supersymmetric effective actions, in proceedings of the 7th International Workshop on Supersymmetries and Quantum Symmetries (SQS'07), Dubna, Russia, 30 July-4 August 2007, pp. 267-274 [INSPIRE].

[29] K. Papadodimas, Topological Anti-Topological Fusion in Four-Dimensional Superconformal Field Theories, JHEP 08 (2010) 118 [arXiv:0910.4963] [INSPIRE].

[30] P. Argyres, M. Lotito, Y. Lü and M. Martone, Geometric constraints on the space of $\mathcal{N}=2$ SCFTs. Part I: physical constraints on relevant deformations, JHEP 02 (2018) 001 [arXiv: 1505.04814] [INSPIRE].

[31] P.C. Argyres, M. Lotito, Y. Lü and M. Martone, Geometric constraints on the space of $\mathcal{N}=2$ SCFTs. Part II: construction of special Kähler geometries and RG flows, JHEP 02 (2018) 002 [arXiv: 1601.00011] [INSPIRE].

[32] P.C. Argyres, M. Lotito, Y. Lü and M. Martone, Expanding the landscape of $\mathcal{N}=2$ rank 1 SCFTs, JHEP 05 (2016) 088 [arXiv: 1602.02764] [INSPIRE].

[33] P. Argyres, M. Lotito, Y. Lü and M. Martone, Geometric constraints on the space of $\mathcal{N}=2$ SCFTs. Part III: enhanced Coulomb branches and central charges, JHEP 02 (2018) 003 [arXiv: 1609.04404] [INSPIRE].

[34] P.C. Argyres and M.R. Douglas, New phenomena in SU(3) supersymmetric gauge theory, Nucl. Phys. B 448 (1995) 93 [hep-th/9505062] [INSPIRE].

[35] P.C. Argyres, M.R. Plesser, N. Seiberg and E. Witten, New $N=2$ superconformal field theories in four-dimensions, Nucl. Phys. B 461 (1996) 71 [hep-th/9511154] [INSPIRE]. 
[36] E. Gerchkovitz, J. Gomis and Z. Komargodski, Sphere Partition Functions and the Zamolodchikov Metric, JHEP 11 (2014) 001 [arXiv:1405.7271] [INSPIRE].

[37] C. Beem, M. Lemos, P. Liendo, W. Peelaers, L. Rastelli and B.C. van Rees, Infinite Chiral Symmetry in Four Dimensions, Commun. Math. Phys. 336 (2015) 1359 [arXiv:1312.5344] [INSPIRE].

[38] D. Jafferis, B. Mukhametzhanov and A. Zhiboedov, Conformal Bootstrap At Large Charge, JHEP 05 (2018) 043 [arXiv: 1710.11161] [INSPIRE].

[39] J.D. Gibbon and M. Tabor, On the one- and two-dimensional Toda lattices and the Painlevé property, J. Math. Phys. 26 (1985) 1956.

[40] Y. Luke, The Special Functions and their Approximations. Volume I, Academic Press, New York U.S.A. (1969), p. 32.

[41] M. Dine and N. Seiberg, Comments on higher derivative operators in some SUSY field theories, Phys. Lett. B 409 (1997) 239 [hep-th/9705057] [INSPIRE].

[42] B. de Wit, M. Roček and S. Vandoren, Hypermultiplets, hyperKähler cones and quaternion Kähler geometry, JHEP 02 (2001) 039 [hep-th/0101161] [INSPIRE].

[43] F. Gonzalez-Rey, M. Roček, S. Wiles, U. Lindström and R. von Unge, Feynman rules in $N=2$ projective superspace: 1. Massless hypermultiplets, Nucl. Phys. B 516 (1998) 426 [hep-th/9710250] [INSPIRE].

[44] J. Wess and J. Bagger, Supersymmetry and supergravity, Princeton University Press, Princeton NJ U.S.A. (1992) [INSPIRE].

[45] D.Z. Freedman and A. Van Proeyen, Supergravity, Cambridge University Press, Cambridge U.K. (2012).

[46] F.A. Dolan and H. Osborn, On short and semi-short representations for four-dimensional superconformal symmetry, Annals Phys. 307 (2003) 41 [hep-th/0209056] [INSPIRE].

[47] C. Cordova, T.T. Dumitrescu and K. Intriligator, Multiplets of Superconformal Symmetry in Diverse Dimensions, JHEP 03 (2019) 163 [arXiv:1612.00809] [INSPIRE].

[48] T.T. Dumitrescu and N. Seiberg, Supercurrents and Brane Currents in Diverse Dimensions, JHEP 07 (2011) 095 [arXiv: 1106.0031] [INSPIRE].

[49] N. Drukker, D.R. Morrison and T. Okuda, Loop operators and S-duality from curves on Riemann surfaces, JHEP 09 (2009) 031 [arXiv:0907.2593] [INSPIRE].

[50] O. Aharony, N. Seiberg and Y. Tachikawa, Reading between the lines of four-dimensional gauge theories, JHEP 08 (2013) 115 [arXiv: 1305.0318] [INSPIRE].

[51] D.M. Hofman and N. Iqbal, Goldstone modes and photonization for higher form symmetries, SciPost Phys. 6 (2019) 006 [arXiv: 1802.09512] [INSPIRE].

[52] N. Evans, T.R. Morris and O.J. Rosten, Gauge invariant regularization in the AdS/CFT correspondence and ghost D-branes, Phys. Lett. B 635 (2006) 148 [hep-th/0601114] [INSPIRE].

[53] T. Okuda and T. Takayanagi, Ghost D-branes, JHEP 03 (2006) 062 [hep-th/0601024] [INSPIRE].

[54] R. Dijkgraaf, B. Heidenreich, P. Jefferson and C. Vafa, Negative Branes, Supergroups and the Signature of Spacetime, JHEP 02 (2018) 050 [arXiv:1603.05665] [INSPIRE]. 
[55] M. Buican and Z. Laczko, Nonunitary Lagrangians and unitary non-Lagrangian conformal field theories, Phys. Rev. Lett. 120 (2018) 081601 [arXiv:1711.09949] [INSPIRE].

[56] G. Festuccia and N. Seiberg, Rigid Supersymmetric Theories in Curved Superspace, JHEP 06 (2011) 114 [arXiv: 1105.0689] [inSPIRE].

[57] T.T. Dumitrescu, G. Festuccia and N. Seiberg, Exploring Curved Superspace, JHEP 08 (2012) 141 [arXiv:1205.1115] [INSPIRE].

[58] S. Hellerman, D. Orlando and S. Reffert, String theory of the Omega deformation, JHEP 01 (2012) 148 [arXiv:1106.0279] [INSPIRE].

[59] E.W. Barnes, The Theory of the Double Gamma Function, Phil. Trans. Roy. Soc. Lond. A 196 (1901) 265.

[60] N.A. Nekrasov, Seiberg-Witten prepotential from instanton counting, Adv. Theor. Math. Phys. 7 (2003) 831 [hep-th/0206161] [INSPIRE].

[61] L.F. Alday, D. Gaiotto and Y. Tachikawa, Liouville Correlation Functions from Four-dimensional Gauge Theories, Lett. Math. Phys. 91 (2010) 167 [arXiv:0906.3219] [INSPIRE]. 